\title{
A DERIVATIVE ARRAY APPROACH FOR LINEAR SECOND ORDER DIFFERENTIAL-ALGEBRAIC SYSTEMS*
}

\author{
LENA SCHOLZ
}

\begin{abstract}
We discuss the solution of linear second order differential-algebraic equations (DAEs) with variable coefficients. Since index reduction and order reduction for higher order, higher index differential-algebraic systems do not commute, appropriate index reduction methods for higher order DAEs are required. We present an index reduction method based on derivative arrays that allows to determine an equivalent second order system of lower index in a numerical computable way. For such an equivalent second order system, an appropriate order reduction method allows the formulation of a suitable first order DAE system of low index that has the same solution components as the original second order system.
\end{abstract}

Key words. Differential-algebraic equation, Second order system, Index reduction, Order reduction, Strangeness index, Strangeness-free system.

AMS subject classifications. 65L80, 65L05, 34A09, 34A12.

1. Introduction. We study linear second order differential-algebraic equations of the form

$$
M(t) \ddot{x}+C(t) \dot{x}+K(t) x=f(t), \quad t \in \mathbb{I},
$$

where $M, C, K \in C\left(\mathbb{I}, \mathbb{C}^{m \times n}\right)$ and $f \in C\left(\mathbb{I}, \mathbb{C}^{m}\right)$ are sufficiently smooth functions on a compact interval $\mathbb{I} \subseteq \mathbb{R}$ with initial conditions

$$
x\left(t_{0}\right)=x_{0} \in \mathbb{C}^{n}, \quad \dot{x}\left(t_{0}\right)=\dot{x}_{0} \in \mathbb{C}^{n} \quad \text { for } t_{0} \in \mathbb{I} .
$$

Here, $C^{k}\left(\mathbb{I}, \mathbb{C}^{m \times n}\right)$ denotes the set of $k$-times continuously differentiable functions from the interval $\mathbb{I}$ to the vector space $\mathbb{C}^{m \times n}$ of complex $m \times n$ matrices. When $n=1$, we use $\mathbb{C}^{m}$ instead of $\mathbb{C}^{m \times 1}$. Systems of this form naturally arise in many technical applications as, e.g., in the simulation of electrical circuits $[8,9]$ or mechanical multibody systems $[6,13]$.

For the numerical (as well as analytical) solution, second order systems of the form (1.1) are usually transformed into first order systems by introducing new variables for the derivatives, as is the common practice in the classical theory of ordinary

\footnotetext{
${ }^{*}$ Received by the editors on November 5, 2008. Accepted for publication on January 31, 2011. Handling Editor: Bryan Shader.

${ }^{\dagger}$ Institut für Mathematik, Technische Universität Berlin, Straße des 17. Juni 136, D-10623 Berlin, Germany (lscholz@math.tu-berlin.de). Supported by DFG Research Center MATHEON, Mathematics for key technologies in Berlin.
} 
differential equations. For DAEs, however, the classical order reduction approach has to be performed with great care, since it may lead to a number of mathematical difficulties as has been discussed in several publications; see [1, 5, 12, 14, 15]. Moreover, the numerical solution of DAEs usually requires the reformulation of higher index DAEs as an equivalent system of lower index to be able to use standard integration methods suited for DAEs; see $[2,10,11]$. Therefore, the numerical solution of linear second order differential-algebraic systems of the form (1.1) typically requires the reduction to a first order system on the one hand and an index reduction for higher index systems on the other hand. But, for high order high index differential-algebraic systems, the order reduction and index reduction do not commute as can be seen in the following example.

EXAMPLE 1.1. We consider the linear second order system

$(1.3)\left[\begin{array}{lll}t & 0 & 0 \\ 0 & 1 & 1 \\ 0 & t & t\end{array}\right]\left[\begin{array}{l}\ddot{x}_{1} \\ \ddot{x}_{2} \\ \ddot{x}_{3}\end{array}\right]+\left[\begin{array}{lll}1 & 0 & 0 \\ 0 & 0 & 0 \\ 0 & 0 & 0\end{array}\right]\left[\begin{array}{l}\dot{x}_{1} \\ \dot{x}_{2} \\ \dot{x}_{3}\end{array}\right]+\left[\begin{array}{ccc}1 & 0 & 0 \\ 0 & 1 & 0 \\ 0 & 1+t & 1\end{array}\right]\left[\begin{array}{l}x_{1} \\ x_{2} \\ x_{3}\end{array}\right]=\left[\begin{array}{c}f_{1} \\ f_{2} \\ f_{3}\end{array}\right]$,

for $t \in\left[t_{0}, t_{1}\right]$ with $t_{0}>0$. System (1.3) has the unique solution components

$$
\left[\begin{array}{l}
x_{2} \\
x_{3}
\end{array}\right]=\left[\begin{array}{c}
f_{2}-\ddot{f}_{3}+t \ddot{f}_{2}+2 \dot{f}_{2} \\
f_{3}-(1+t) f_{2}+\ddot{f}_{3}-t \ddot{f}_{2}-2 \dot{f}_{2}
\end{array}\right]
$$

and $x_{1}$ is the unique solution of the second order ordinary differential equation $t \ddot{x}_{1}+$ $\dot{x}_{1}+x_{1}=f_{1}$ for some given initial values $x_{1}\left(t_{0}\right)=x_{1,0}$ and $\dot{x}_{1}\left(t_{0}\right)=\dot{x}_{1,0}$. Hence, the minimum requirement for the existence of a continuous solution is that $f_{1}$ is continuous, and $f_{2}$ and $f_{3}$ are twice continuously differentiable (corresponding to a strangeness index of $\mu=2$ ). The classical order reduction for the second order system (1.3) yields a first order system of the form

$$
\left[\begin{array}{ccc|ccc}
t & 0 & 0 & 0 & 0 & 0 \\
0 & 1 & 1 & 0 & 0 & 0 \\
0 & t & t & 0 & 0 & 0 \\
\hline 0 & 0 & 0 & 1 & 0 & 0 \\
0 & 0 & 0 & 0 & 1 & 0 \\
0 & 0 & 0 & 0 & 0 & 1
\end{array}\right]\left[\begin{array}{c}
\dot{v}_{1} \\
\dot{v}_{2} \\
\dot{v}_{3} \\
\dot{x}_{1} \\
\dot{x}_{2} \\
\dot{x}_{3}
\end{array}\right]+\left[\begin{array}{ccc|ccc}
1 & 0 & 0 & 1 & 0 & 0 \\
0 & 0 & 0 & 0 & 1 & 0 \\
0 & 0 & 0 & 0 & 1+t & 1 \\
\hline-1 & 0 & 0 & 0 & 0 & 0 \\
0 & -1 & 0 & 0 & 0 & 0 \\
0 & 0 & -1 & 0 & 0 & 0
\end{array}\right]\left[\begin{array}{c}
v_{1} \\
v_{2} \\
v_{3} \\
x_{1} \\
x_{2} \\
x_{3}
\end{array}\right]=\left[\begin{array}{c}
f_{1} \\
f_{2} \\
f_{3} \\
0 \\
0 \\
0
\end{array}\right] .
$$

In comparison to the solution of (1.3), this system has the additional solution components

$$
\left[\begin{array}{l}
v_{2} \\
v_{3}
\end{array}\right]=\left[\begin{array}{c}
\dot{f}_{2}-f_{3}^{(3)}+t f_{2}^{(3)}+3 \ddot{f}_{2} \\
\dot{f}_{3}-(t+1) \dot{f}_{2}-f_{2}+f_{3}^{(3)}-t f_{2}^{(3)}-3 \ddot{f}_{2}
\end{array}\right]
$$

i.e., the third derivative of the inhomogeneity is required (the system is of strangeness 
index $\tilde{\mu}=3$; see [11]). On the other hand, system (1.3) is equivalent to the system

$$
\begin{aligned}
& {\left[\begin{array}{lll}
t & 0 & 0 \\
0 & 0 & 0 \\
0 & 0 & 0
\end{array}\right]\left[\begin{array}{l}
\ddot{x}_{1} \\
\ddot{x}_{2} \\
\ddot{x}_{3}
\end{array}\right]+\left[\begin{array}{lll}
1 & 0 & 0 \\
0 & 0 & 0 \\
0 & 0 & 0
\end{array}\right]\left[\begin{array}{l}
\dot{x}_{1} \\
\dot{x}_{2} \\
\dot{x}_{3}
\end{array}\right]+\left[\begin{array}{lll}
1 & 0 & 0 \\
0 & 1 & 0 \\
0 & 0 & 1
\end{array}\right]\left[\begin{array}{l}
x_{1} \\
x_{2} \\
x_{3}
\end{array}\right]} \\
& =\left[\begin{array}{c}
f_{1} \\
f_{2}-\ddot{f}_{3}+\left(t f_{2}\right)^{\prime \prime} \\
f_{3}-t f_{2}-f_{2}+\ddot{f}_{3}-\left(t f_{2}\right)^{\prime \prime}
\end{array}\right],
\end{aligned}
$$

a decoupled system of two algebraic equations and one differential equation. Now, introducing only $v_{1}=\dot{x}_{1}$ as new variable, we get the first order system

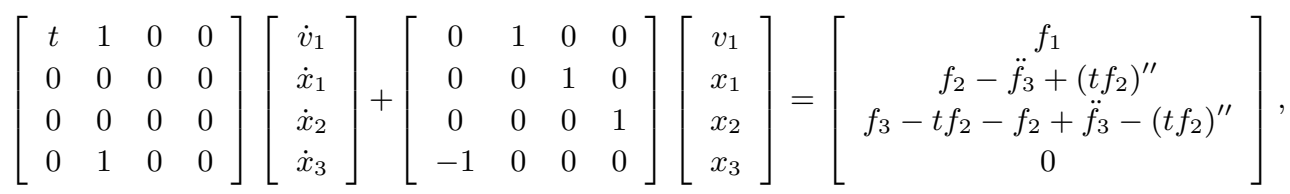

which is also a decoupled system of algebraic and differential equations and no further smoothness requirements are imposed.

Further examples are presented in [12]. They show that the classical approach of introducing the derivatives of the unknown vector-valued function $x(t)$ as new variables may lead to higher smoothness requirements for the inhomogeneity $f(t)$ to ensure the existence of a solution that can even cause the loss of solvability of the system. By introducing only some new variables, however, this difficulty can be circumvented. An index reduction method and condensed forms for linear high order differential-algebraic systems are introduced in [12], which allow an identification of those higher order derivatives of variables that can be replaced to obtain a first order system without changing the smoothness requirements. But, the computation of this condensed form is not feasible for numerical solution methods as it involves the derivatives of computed transformation matrices. However, since the standard way to obtain a strangeness-free first order formulation-first introducing new variables for the derivatives to transform the system into a first order system and then applying the usual index reduction procedures to the first order system-can fail due to a possible increase in the index, at first an index reduction of the higher order system should be used, which is followed by an appropriate order reduction to obtain a suitable strangeness-free first order formulation. Recently, it has been shown in $[14,17]$ that also the direct discretization of the second order system may yield better numerical results and is able to prevent certain numerical difficulties as the failure of numerical methods; see also $[1,2,16]$.

In this paper, we will present a new index reduction method for linear second order differential-algebraic systems of the form (1.1), based on the derivatives of the coefficient matrices $M(t), C(t)$ and $K(t)$, that allows the computation of an equivalent 
system of low index and in a second step also the formulation of a corresponding trimmed first order formulation. At first, in Section 2, we present the basic results of the analysis of linear second order differential-algebraic equations as derived in [12], including a new condensed form that allows one to read off the characteristic invariants of the differential-algebraic system. In Section 3, we introduce the derivative array approach which enables us to transform the linear second order system (1.1) into an equivalent strangeness-free second order system with the same solution set in a numerically computable way. Further, in Section 4, we present a trimmed first order formulation for linear strangeness-free second order systems. Throughout this paper, for ease of presentation, we restrict to linear second order systems since they are most frequently used in practical applications. However, all presented ideas can also be extended to arbitrary linear $k$-th order systems and to nonlinear systems; see [18].

2. Condensed forms for linear second order DAEs. In the following, we present the main results of the analysis of linear second order differential-algebraic systems of the form (1.1) as derived in [12]. The condensed forms given in [12] are used to derive the relationships between the global invariants of the triple of matrix-valued functions $(M, C, K)$ and the local invariants of the derivative array as presented in Section 3. To derive condensed forms for triples $(M, C, K)$ of matrix-valued functions we need an appropriate equivalence relation.

Definition 2.1. Two triples $\left(M_{1}, C_{1}, K_{1}\right)$ and $\left(M_{2}, C_{2}, K_{2}\right)$ of matrix-valued functions $M_{i}, C_{i}, K_{i} \in C\left(\mathbb{I}, \mathbb{C}^{m \times n}\right), i=1,2$ are called globally equivalent if there exist pointwise nonsingular matrix-valued functions $P \in C\left(\mathbb{I}, \mathbb{C}^{m \times m}\right)$ and $Q \in C^{2}\left(\mathbb{I}, \mathbb{C}^{n \times n}\right)$ such that

$$
M_{2}=P M_{1} Q, \quad C_{2}=2 P M_{1} \dot{Q}+P C_{1} Q, \quad K_{2}=P M_{1} \ddot{Q}+P C_{1} \dot{Q}+P K_{1} Q .
$$

For equivalent matrix triples we write $\left(M_{1}, C_{1}, K_{1}\right) \sim\left(M_{2}, C_{2}, K_{2}\right)$.

Considering the action of the equivalence relation (2.1) locally at a fixed point $\hat{t} \in \mathbb{I}$, we take into account that for given matrices $\hat{P}, \hat{Q}, \hat{R}_{1}$ and $\hat{R}_{2}$ of appropriate size, using Hermite interpolation, we can always find matrix-valued functions $P$ and $Q$, such that at a given value $t=\hat{t}$ we have $P(\hat{t})=\hat{P}, Q(\hat{t})=\hat{Q}, \dot{Q}(\hat{t})=\hat{R}_{1}$ and $\ddot{Q}(\hat{t})=\hat{R}_{2}$. Therefore, we can define local equivalence of matrix triples in the following way.

Definition 2.2. Two matrix triples $\left(M_{1}, C_{1}, K_{1}\right)$ and $\left(M_{2}, C_{2}, K_{2}\right)$ with $M_{i}$, $C_{i}, K_{i} \in \mathbb{C}^{m \times n}, i=1,2$, are called locally equivalent if there exist nonsingular matrices $P \in \mathbb{C}^{m \times m}$ and $Q \in \mathbb{C}^{n \times n}$, and matrices $R_{1}, R_{2} \in \mathbb{C}^{n \times n}$ such that

$$
M_{2}=P M_{1} Q, \quad C_{2}=2 P M_{1} R_{1}+P C_{1} Q, \quad K_{2}=P M_{1} R_{2}+P C_{1} R_{1}+P K_{1} Q .
$$

Again, we write $\left(M_{1}, C_{1}, K_{1}\right) \sim\left(M_{2}, C_{2}, K_{2}\right)$ if the context is clear. 
It has be shown in [15] that the relations (2.1) and (2.2) are equivalence relations on the set of triples of matrix-valued functions, and on the set of triples of matrices, respectively. For a linear second order differential-algebraic system of the form (1.1), a condensed form under local equivalence transformation (2.2) of the corresponding matrix triple $(M(\hat{t}), C(\hat{t}), K(\hat{t}))$ at a fixed point $\hat{t} \in \mathbb{I}$ has been derived in $[12,15]$. This local condensed form allows to characterize second order differential-algebraic systems locally by their purely first and second order differential parts of size $d^{(1)}$ and $d^{(2)}$, by their algebraic part of size $a$, by undetermined and redundant parts of size $u$ and $v$, and by the strangeness parts of size $s^{(M C K)}, s^{(M C)}, s^{(M K)}$ and $s^{(C K)}$ due to the different possible couplings between the matrices $M, C$, and $K$. The quantities $s^{(M C K)}, s^{(M C)}, s^{(M K)}, s^{(C K)}, d^{(2)}, d^{(1)}, a, v$ and $u$ are called the local characteristic values of the linear second order DAE (1.1). These local characteristic values are invariant under the equivalence relation (2.2) and can be expressed in terms of ranks of matrices and dimensions of column spaces.

Lemma 2.3. [12, 15] Let $M, C, K \in \mathbb{C}^{m \times n}$ and let

$V_{1}$ be a basis of $\operatorname{kernel}\left(M^{H}\right)$,

$V_{2}$ be a basis of $\operatorname{kernel}(M)$,

$V_{3}$ be a basis of $\operatorname{kernel}\left(M^{H}\right) \cap \operatorname{kernel}\left(C^{H}\right)$,

$V_{4}$ be a basis of $\operatorname{kernel}(M) \cap \operatorname{kernel}\left(V_{1}^{H} C\right)$.

Then, the quantities

$$
\begin{array}{ll}
r=\operatorname{rank}(M) & \text { (rank of } M) \\
a=\operatorname{rank}\left(V_{3}^{H} K V_{4}\right) & \text { (algebraic part) } \\
s^{(M C K)}=\operatorname{dim}\left(\operatorname{range}\left(M^{H}\right) \cap \operatorname{range}\left(C^{H} V_{1}\right) \cap \operatorname{range}\left(K^{H} V_{3}\right)\right) & \text { (strangeness of } M, C, K) \\
s^{(C K)}=\operatorname{rank}\left(V_{3}^{H} K V_{2}\right)-a & \text { (strangeness of } C, K) \\
d^{(1)}=\operatorname{rank}\left(V_{1}^{H} C V_{2}\right)-s^{(C K)} & \text { (1st-order diff. part) } \\
s^{(M C)}=\operatorname{rank}\left(V_{1}^{H} C\right)-s^{(M C K)}-s^{(C K)}-d^{(1)} & \text { (strangeness of } M, C) \\
s^{(M K)}=\operatorname{rank}\left(V_{3}^{H} K\right)-a-s^{(M C K)}-s^{(C K)} & \text { (strangeness of } M, K) \\
d^{(2)}=r-s^{(M C K)}-s^{(M C)}-s^{(M K)} & \text { (2nd-order diff. part) } \\
v=m-r-2 s^{(C K)}-d^{(1)}-2 s^{(M C K)}-s^{(M C)}-a-s^{(M K)} & \text { (vanishing equations) } \\
u=n-r-s^{(C K)}-d^{(1)}-a & \text { (undetermined part) }
\end{array}
$$

are invariant under the local equivalence relation (2.2).

For triples $(M(t), C(t), K(t))$ of matrix-valued functions, we can compute the local condensed form at any fixed value $\hat{t} \in \mathbb{I}$ and determine the local characteristic quantities so that we obtain functions

$$
r, a, d^{(2)}, d^{(1)}, s^{(M C K)}, s^{(C K)}, s^{(M C)}, s^{(M K)}, u, v: \mathbb{I} \longrightarrow \mathbb{N}_{0} .
$$


Assuming that these functions are constant over the interval $\mathbb{I}$, i.e.,

$$
\begin{gathered}
r(t) \equiv r, \quad a(t) \equiv a, \quad d^{(1)}(t) \equiv d^{(1)}, \quad s^{(M C K)}(t) \equiv s^{(M C K)} \\
s^{(C K)}(t) \equiv s^{(C K)}, \quad s^{(M C)}(t) \equiv s^{(M C)}, \quad s^{(M K)}(t) \equiv s^{(M K)}, \quad \text { for all } t \in \mathbb{I},
\end{gathered}
$$

(yielding that also $d^{(2)}(t), u(t)$ and $v(t)$ are constant in $\mathbb{I}$ due to Lemma 2.3) a global condensed form for triples of matrix-valued functions under global equivalence transformations (2.1) has been derived in $[12,15]$ (see Lemma A.1 in Appendix). Using this global condensed form a stepwise index reduction procedure incorporating differentiations of equations and eliminations of certain coupling parts in the differentialalgebraic system finally yields a so-called strangeness-free second order system of DAEs where the strangeness parts have vanished. We call the required number of steps $\mu$ in the index reduction procedure the strangeness index or s-index of the second order system of DAEs (1.1). For a more detailed description of the index reduction procedure, see also $[12,19]$.

THEOREM 2.4. Consider the linear second order system (1.1), suppose that the regularity conditions (2.3) hold, and let $\mu$ be the strangeness index of (1.1). If $f \in$ $C^{\mu}\left(\mathbb{I}, \mathbb{C}^{m}\right)$, then system (1.1) is equivalent (in the sense that there is a one-to-one correspondence between the solution sets) to a strangeness-free system of second order differential-algebraic equations of the form

$$
\begin{array}{rlrl}
\ddot{\tilde{x}}_{1}+\tilde{C}_{11}(t) \dot{\tilde{x}}_{1}+\tilde{C}_{14}(t) \dot{\tilde{x}}_{4}+\tilde{K}_{11}(t) \tilde{x}_{1}+\tilde{K}_{12}(t) \tilde{x}_{2}+\tilde{K}_{14}(t) \tilde{x}_{4} & =\tilde{f}_{1}(t), & & \left(d_{\mu}^{(2)}\right) \\
\dot{\tilde{x}}_{2}+\tilde{K}_{21}(t) \tilde{x}_{1}+\tilde{K}_{22}(t) \tilde{x}_{2}+\tilde{K}_{24}(t) \tilde{x}_{4} & =\tilde{f}_{2}(t), & & \left(d_{\mu}^{(1)}\right) \\
\tilde{x}_{3} & =\tilde{f}_{3}(t), & \left(a_{\mu}\right) \\
0 & =\tilde{f}_{4}(t), & \left(v_{\mu}\right)
\end{array}
$$

where the inhomogeneity $\tilde{f}:=\left[\tilde{f}_{1}^{H}, \ldots, \tilde{f}_{4}^{H}\right]^{H}$ is determined by $f^{(0)}, \ldots, f^{(\mu)}$. In particular, $d_{\mu}^{(2)}, d_{\mu}^{(1)}$ and $a_{\mu}$ are respectively the number of second order differential, the number of first order differential, and the number of algebraic components of the unknown $\tilde{x}:=\left[\tilde{x}_{1}^{H}, \ldots, \tilde{x}_{4}^{H}\right]^{H}$, while $u_{\mu}$ is the dimension of the undetermined vector $\tilde{x}_{4}$, and $v_{\mu}$ is the number of conditions in the last equation.

Proof. See [12] or [19].

Using the strangeness-free form (2.4) we can analyze existence and uniqueness of solutions and consistency of initial conditions for linear second order differentialalgebraic systems (1.1), see [12, 15]. Further, the strangeness-free form (2.4) allows the identification of those second order derivatives of variables that can be replaced to obtain a first order system that is strangeness-free without increasing the index (see also Section 4). 
REMARK 2.5. In $[18,19]$, we use a slightly different stepwise index reduction procedure compared to $[12,15]$. In $[12,15]$ one or two differentiations of equations are required for one index reduction step, depending on the occurrence of strangeness blocks, since all strangeness parts are completely eliminated in every reduction step. In this way, the index definition does not correspond to the differentiability requirements for the right hand side. In our approach described in [18, 19], the right-hand side is only differentiated once in each elimination step before the system is again transformed to global condensed form such that the strangeness index corresponds to the differentiability requirements for the right hand side, which is the case for all general index concepts.

The sequence of characteristic values, obtained during the stepwise index reduction procedure, can also be characterized recursively in terms of ranks of block matrices of the matrix triple.

LEMMA 2.6. Let the functions $M, C, K \in C\left(\mathbb{I}, \mathbb{C}^{m \times n}\right)$ be sufficiently smooth and let the strangeness index $\mu$ be well-defined. Further, let the process leading to Theorem 2.4 yield a sequence $\left(M^{<i>}, C^{<i>}, K^{<i>}\right), i \in \mathbb{N}_{0}$, with $\left(M^{<0>}, C^{<0>}, K^{<0>}\right)=$ $(M, C, K)$ and characteristic values $\left(r_{i}, d_{i}^{(1)}, a_{i}, s_{i}^{(M C K)}, s_{i}^{(M C)}, s_{i}^{(M K)}, s_{i}^{(C K)}, u_{i}, v_{i}\right)$ according to Lemma 2.3. The triple $\left(M^{<i>}, C^{<i>}, K^{<i>}\right)$ of matrix-valued functions is globally equivalent to the triple

$\left(\begin{array}{ccccccccc}I_{s_{i}^{(M C K)}} & 0 & 0 & 0 & 0 & 0 & 0 & 0 & 0 \\ 0 & I_{s_{i}^{(M C)}} & 0 & 0 & 0 & 0 & 0 & 0 & 0 \\ 0 & 0 & I_{s_{i-1}} & 0 & 0 & 0 & 0 & 0 & 0 \\ 0 & 0 & 0 & I_{s_{i}} & 0 & 0 & 0 & 0 & 0 \\ 0 & 0 & 0 & 0 & I_{d_{i}^{(2)}} & 0 & 0 & 0 & 0 \\ 0 & 0 & 0 & 0 & 0 & 0 & 0 & 0 & 0 \\ 0 & 0 & 0 & 0 & 0 & 0 & 0 & 0 & 0 \\ 0 & 0 & 0 & 0 & 0 & 0 & 0 & 0 & 0 \\ 0 & 0 & 0 & 0 & 0 & 0 & 0 & 0 & 0 \\ 0 & 0 & 0 & 0 & 0 & 0 & 0 & 0 & 0 \\ 0 & 0 & 0 & 0 & 0 & 0 & 0 & 0 & 0 \\ 0 & 0 & 0 & 0 & 0 & 0 & 0 & 0 & 0 \\ 0 & 0 & 0 & 0 & 0 & 0 & 0 & 0 & 0 \\ 0 & 0 & 0 & 0 & 0 & 0 & 0 & 0 & 0 \\ 0 & 0 & 0 & 0 & 0 & 0 & 0 & 0 & 0\end{array}\right]$,




\begin{tabular}{|c|c|c|c|c|c|c|c|c|c|}
\hline 0 & 0 & $C_{13}^{<i>}$ & $C_{14}^{<i>}$ & $C_{15}^{<i>}$ & 0 & 0 & $C_{18}^{<i>}$ & $C_{19}^{<i>}$ & \\
\hline 0 & 0 & $C_{23}^{<i>}$ & $C_{24}^{<i>}$ & $C_{25}^{<i>}$ & 0 & 0 & $C_{28}^{<i>}$ & $C_{29}^{<i>}$ & \\
\hline 0 & 0 & $C_{33}^{<i>}$ & $C_{34}^{<i>}$ & $C_{35}^{<i>}$ & 0 & 0 & $C_{38}^{<i>}$ & $C_{39}^{<i>}$ & \\
\hline 0 & 0 & $C_{43}^{<i>}$ & $C_{44}^{<i>}$ & $C_{45}^{<i>}$ & 0 & 0 & $C_{48}^{<i>}$ & $C_{49}^{<i>}$ & \\
\hline 0 & 0 & $C_{53}^{<i>}$ & $C_{54}^{<i>}$ & $C_{55}^{<i>}$ & 0 & 0 & $C_{58}^{<i>}$ & $C_{59}^{<i>}$ & \\
\hline 0 & 0 & 0 & 0 & 0 & $I_{s}(C K)$ & 0 & 0 & 0 & \\
\hline 0 & 0 & 0 & 0 & 0 & ${ }^{i} 0$ & $I_{d_{i}^{(1)}}$ & 0 & 0 & \\
\hline$I_{s_{i}(M C K)}$ & 0 & 0 & 0 & 0 & 0 & 0 & 0 & 0 & , \\
\hline 0 & $I_{s_{i}^{(M C)}}$ & 0 & 0 & 0 & 0 & 0 & 0 & 0 & \\
\hline 0 & ${ }^{2} 0$ & 0 & 0 & 0 & 0 & 0 & 0 & 0 & \\
\hline 0 & 0 & 0 & 0 & 0 & 0 & 0 & 0 & 0 & \\
\hline 0 & 0 & 0 & 0 & 0 & 0 & 0 & 0 & 0 & \\
\hline 0 & 0 & 0 & 0 & 0 & 0 & 0 & 0 & 0 & \\
\hline 0 & 0 & 0 & 0 & 0 & 0 & 0 & 0 & 0 & \\
\hline 0 & 0 & 0 & 0 & 0 & 0 & 0 & 0 & 0 & \\
\hline 0 & $K_{12}^{<i>}$ & 0 & 0 & $K_{15}^{<i>}$ & 0 & $K_{17}^{<i>}$ & 0 & $K_{19}^{<i>}$ & $s_{i}^{(M C K)}$ \\
\hline 0 & $K_{22}^{<i>}$ & 0 & 0 & $K_{25}^{<i>}$ & 0 & $K_{27}^{<i>}$ & 0 & $K_{29}^{<i>}$ & $s_{i}^{(M C)}$ \\
\hline 0 & $K_{32}^{<i>}$ & 0 & 0 & $K_{35}^{<i>}$ & 0 & $K_{37}^{<i>}$ & 0 & $K_{39}^{<i>}$ & $s_{i-1}$ \\
\hline 0 & $K_{42}^{<i>}$ & 0 & 0 & $K_{45}^{<i>}$ & 0 & $K_{47}^{<i>}$ & 0 & $K_{49}^{<i>}$ & $s_{i}$ \\
\hline 0 & $K_{52}^{<i>}$ & 0 & 0 & $K_{55}^{<i>}$ & 0 & $K_{57}^{<i>}$ & 0 & $K_{59}^{<i>}$ & $d_{i}^{(2)}$ \\
\hline 0 & $K_{62}^{<i>}$ & 0 & 0 & $K_{65}^{<i>}$ & 0 & $K_{67}^{<i>}$ & 0 & $K_{69}^{<i>}$ & $s_{i}^{(C K)}$ \\
\hline 0 & $K_{72}^{<i>}$ & 0 & 0 & $K_{75}^{<i>}$ & 0 & $K_{77}^{<i>}$ & 0 & $K_{79}^{<i>}$ & $d_{i}^{(1)}$ \\
\hline 0 & $K_{82}^{<i>}$ & 0 & 0 & $K_{85}^{<i>}$ & 0 & $K_{87}^{<i>}$ & 0 & $K_{89}^{<i>}$ & $s_{i}^{(M C K)}$ \\
\hline 0 & $K_{92}^{<i>}$ & 0 & 0 & $K_{95}^{<i>}$ & 0 & $K_{97}^{<i>}$ & 0 & $K_{99}^{<i>}$ & $s_{i}^{(M C)}$ \\
\hline 0 & 0 & 0 & 0 & 0 & 0 & 0 & $I_{a_{i}}$ & 0 & $a_{i}$ \\
\hline 0 & 0 & 0 & 0 & 0 & $I_{s_{i}^{(C K)}}$ & 0 & 0 & 0 & $s_{i}^{(C K)}$ \\
\hline 0 & 0 & $I_{s_{i-1}}$ & 0 & 0 & 0 & 0 & 0 & 0 & $s_{i-1}$ \\
\hline 0 & 0 & 0 & $I_{s_{i}}$ & 0 & 0 & 0 & 0 & 0 & $s_{i}$ \\
\hline$I_{s_{i}^{(M C K)}}$ & 0 & 0 & 0 & 0 & 0 & 0 & 0 & 0 & $s_{i}^{(M C K)}$ \\
\hline 0 & 0 & 0 & 0 & 0 & 0 & 0 & 0 & 0 & $v_{i}$ \\
\hline
\end{tabular}

where $s_{i}^{(M K)}$ is separated into $s_{i}^{(M K)}=s_{i}+s_{i-1}$ (with $\left.s_{-1}=0\right)$ and the last block columns have size $u_{i}$. We define

$$
\begin{aligned}
\tilde{C}_{1 j}^{<i>} & :=C_{1 j}^{<i>}-K_{8 j}^{<i>}, \quad j=5,9, \\
\tilde{C}_{2 j}^{<i>} & :=C_{2 j}^{<i>}-K_{9 j}^{<i>}, \quad j=5,9, \\
\tilde{K}_{1 j}^{<i>} & :=K_{1 j}^{<i>}-\dot{K}_{8 j}^{<i>}+K_{82}^{<i>} K_{9 j}^{<i>}+K_{87}^{<i>} K_{7 j}^{<i>}, \quad j=2,5,7,9, \\
\tilde{K}_{2 j}^{<i>} & :=K_{2 j}^{<i>}-\dot{K}_{9 j}^{<i>}+K_{92}^{<i>} K_{9 j}^{<i>}+K_{97}^{<i>} K_{7 j}^{<i>}, j=2,5,7,9, \\
\tilde{C}_{1} & :=\left[\begin{array}{lll}
\tilde{C}_{15}^{<i>H} & \tilde{C}_{25}^{<i>H} & C_{35}^{<i>H}
\end{array}\right]^{H}, \\
\tilde{C}_{2} & :=\left[\begin{array}{lll}
\tilde{C}_{19}^{<i>H} & \tilde{C}_{29}^{<i>H} & C_{39}^{<i>H}
\end{array}\right]^{H},
\end{aligned}
$$


as well as

$$
\begin{array}{ll}
k_{0}=d_{0}^{(1)}+s_{0}^{(C K)}, & k_{i+1}=\operatorname{rank} \tilde{C}_{2}, \\
e_{0}=d_{0}^{(1)}+s_{0}^{(M C)}+s_{0}^{(C K)}+s_{0}^{(M C K)}, & e_{i+1}=\operatorname{rank}\left(\left[\begin{array}{ll}
\tilde{C}_{1} & \tilde{C}_{2}
\end{array}\right]\right) .
\end{array}
$$

Then let $U$ and $V$ be nonsingular matrix-valued functions of size $\left(s_{i}^{(M C K)}+s_{i}^{(M C)}+\right.$ $\left.s_{i-1}, s_{i}^{(M C K)}+s_{i}^{(M C)}+s_{i-1}\right)$ and $\left(d_{i}^{(2)}+u_{i}, d_{i}^{(2)}+u_{i}\right)$, respectively, such that

$$
U^{H}\left[\begin{array}{cc}
\tilde{C}_{1} & \tilde{C}_{2}
\end{array}\right] V=\left[\begin{array}{cc}
I_{e_{i+1}} & 0 \\
0 & 0
\end{array}\right]
$$

Further, let $U$ and $V$ be partitioned into

$$
U=\left[\begin{array}{lll}
U_{1} & U_{2} & U_{3}
\end{array}\right], \quad V=\left[\begin{array}{lll}
V_{1} & V_{2} & V_{3}
\end{array}\right]
$$

such that

$$
\left[\begin{array}{c}
U_{1}^{H} \\
U_{2}^{H} \\
U_{3}^{H}
\end{array}\right]\left[\begin{array}{ll}
\tilde{C}_{1} & \tilde{C}_{2}
\end{array}\right]\left[\begin{array}{lll}
V_{1} & V_{2} & V_{3}
\end{array}\right]=\left[\begin{array}{ccc}
I_{e_{i+1}-k_{i+1}} & 0 & 0 \\
0 & I_{k_{i+1}} & 0 \\
0 & 0 & 0
\end{array}\right]
$$

and with a splitting of $V_{3}$ into $V_{3}=\left[\begin{array}{ll}V_{31} & V_{32}\end{array}\right]$ with $V_{31}$ of size $\left(d_{i}^{(2)}+u_{i}, d_{i}^{(2)}-\right.$ $\left.e_{i+1}+k_{i+1}\right)$ and $V_{32}$ of size $\left(d_{i}^{(2)}+u_{i}, u_{i}-k_{i+1}\right)$ we can define

$$
\begin{aligned}
& {\left[\begin{array}{llllll}
K_{1} & K_{2} & K_{3} & K_{4} & K_{5} & K_{6}
\end{array}\right]:=}
\end{aligned}
$$

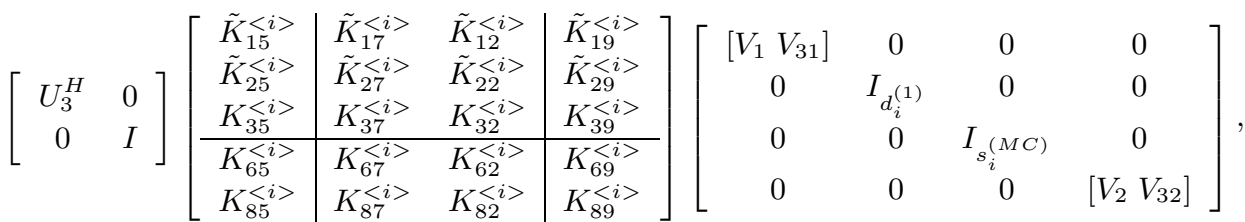

where the identity matrix on the left-hand side is of size $s_{i}^{(C K)}+s_{i}^{(M C K)}$. Further, we define

$$
\begin{array}{ll}
b_{0}=a_{0}, & b_{i+1}=\operatorname{rank}\left(\left[K_{6}\right]\right), \\
p_{0}=a_{0}+s_{0}^{(C K)}, & p_{i+1}=\operatorname{rank}\left(\left[K_{5} K_{6}\right]\right), \\
t_{0}=a_{0}+s_{0}^{(C K)}-s_{0}^{(M K)}, & t_{i+1}=\operatorname{rank}\left(\left[K_{4} K_{5} K_{6}\right]\right), \\
d_{0}=a_{0}+s_{0}^{(C K)}, & d_{i+1}=\operatorname{rank}\left(\left[K_{3} K_{4} K_{5} K_{6}\right]\right), \\
h_{0}=a_{0}+s_{0}^{(C K)}+s_{0}^{(M K)}, & h_{i+1}=\operatorname{rank}\left(\left[K_{2} K_{3} K_{4} K_{5} K_{6}\right]\right), \\
c_{0}=a_{0}+s_{0}^{(M C K)}+s_{0}^{(C K)}+s_{0}^{(M K)}, & c_{i+1}=\operatorname{rank}\left(\left[K_{1} K_{2} K_{3} K_{4} K_{5} K_{6}\right]\right), \\
w_{0}=v_{0}, & w_{i+1}=v_{i+1}-v_{i}, \\
q_{0}=e_{0}, & q_{i+1}=e_{i+1}+c_{i}-s_{i}^{(C K)}-s_{i}^{(M C K)} .
\end{array}
$$


Then we have

$$
\begin{aligned}
r_{i+1} & =r_{i}-s_{i}^{(M C K)}-s_{i}^{(M C)}-s_{i-1}, \\
c_{i+1} & =b_{i+1}+s_{i+1}^{(M C K)}+s_{i+1}^{(C K)}+s_{i+1}^{(M K)}-s_{i}, \\
e_{i+1} & =k_{i+1}+s_{i+1}^{(M C)}+s_{i+1}^{(M C K)} \\
a_{i+1} & =a_{i}+s_{i}^{(C K)}+s_{i}^{(M C K)}+s_{i-1}+b_{i+1} \\
& =c_{0}+\ldots+c_{i+1}-s_{i+1}^{(C K)}-s_{i+1}^{(M K)}-s_{i+1}^{(M C K)}, \\
s_{i+1}^{(M C K)} & =c_{i+1}-h_{i+1}, \\
s_{i+1}^{(M C)} & =e_{i+1}-k_{i+1}-c_{i+1}+h_{i+1}, \\
s_{i+1} & =h_{i+1}-d_{i+1}, \\
s_{i+1}^{(M K)} & =s_{i+1}+s_{i}, \\
s_{i+1}^{(C K)} & =d_{i+1}-b_{i+1}, \\
d_{i+1}^{(2)} & =r_{i+1}-s_{i+1}^{(M C K)}-s_{i+1}^{(M C)}-s_{i+1}^{(M K)}=d_{i}^{(2)}-e_{i+1}+k_{i+1}-s_{i+1}, \\
d_{i+1}^{(1)} & =d_{i}^{(1)}+s_{i}^{(M C)}+k_{i+1}-s_{i+1}^{(C K)} \\
& =q_{0}+\ldots+q_{i+1}-c_{0}-\ldots-c_{i}-s_{i+1}^{(M C K)}-s_{i+1}^{(M C)}-s_{i+1}^{(C K)}, \\
w_{i+1} & =2 s_{i}^{(M C K)}+s_{i}^{(C K)}+s_{i}^{(M C)}+s_{i-1}-e_{i+1}-c_{i+1}, \\
u_{i+1} & =u_{0}-b_{1}-\ldots-b_{i+1}, \\
v_{i+1} & =v_{0}+w_{1}+\ldots+w_{i+1} \\
& =2 s_{i}^{(M C K)}+s_{i}^{(C K)}+s_{i}^{(M C)}+s_{i-1}-e_{i+1}-c_{i+1}+v_{i} .
\end{aligned}
$$

Proof. We omit the proof for ease of presentation. The proof is given in $[18,19]$.

3. Derivative array approach. The algebraic approach described in the previous section allows for the theoretical analysis of linear second order DAEs (1.1), but it cannot be used for the development of numerical methods as neither the inductive process of the reduction to the strangeness-free formulation (2.4) nor the global condensed form is obtained in a way that is feasible for numerical methods. Therefore, we look for other ways to compute the characteristic invariants of a given DAE as well as a canonical form similar to (2.4) in a numerically stable procedure. The basic idea due to Campbell [4] is to differentiate the differential-algebraic equation (1.1) a number of times and put the original DAE and its derivatives into a large system. Then purely local invariants can be constructed via local equivalence transformations, which allow to determine the global invariants including the strangeness index, wherever they are defined. Furthermore, it is also possible to derive a strangeness-free formulation using only local informations. 
In the following, we consider matrix-valued functions $M, C, K \in C\left(\mathbb{I}, \mathbb{C}^{m, n}\right)$ that are sufficiently smooth and we assume that the strangeness index $\mu$ is well-defined, i.e., the ranks are constant in the considered interval and none of the invariant values changes its value during the process. This can always be achieved by going to smaller intervals, since there always exist open intervals $\mathbb{I}_{j} \subseteq \mathbb{I}, j \in \mathbb{N}$ with $\overline{\bigcup_{j \in \mathbb{N}} \mathbb{I}_{j}}=\mathbb{I}$, $\mathbb{I}_{i} \cap \mathbb{I}_{j}=\emptyset$ for $i \neq j$ such that the constant rank assumption holds for all $t \in \mathbb{I}_{j}$, $j \in \mathbb{N}$, and the following construction can be applied separately for each interval $\mathbb{I}_{j}$; for example, see [11]. Differentiating the differential-algebraic equation (1.1) and putting the original DAE and its derivatives up to a sufficiently high order into a large system, we obtain the derivative array associated with the linear second order DAE (1.1) of the form

$$
\mathcal{M}_{l}(t) \ddot{z}_{l}+\mathcal{L}_{l}(t) \dot{z}_{l}+\mathcal{N}_{l}(t) z_{l}=g_{l}(t), \quad l \in \mathbb{N}_{0},
$$

where $\mathcal{M}_{l}, \mathcal{L}_{l}, \mathcal{N}_{l}, z_{l}$ and $g_{l}$ are defined by

$$
\begin{aligned}
{\left[\mathcal{M}_{l}\right]_{i, j} } & :=\left(\begin{array}{l}
i \\
j
\end{array}\right) M^{(i-j)}+\left(\begin{array}{c}
i \\
j+1
\end{array}\right) C^{(i-j-1)}+\left(\begin{array}{c}
i \\
j+2
\end{array}\right) K^{(i-j-2)}, \quad i, j=0, \ldots, l, \\
{\left[\mathcal{L}_{l}\right]_{i, j} } & := \begin{cases}C^{(i)}+i K^{(i-1)} & \text { for } i=0, \ldots, l, j=0, \\
0 & \text { otherwise },\end{cases} \\
(3.2)\left[\mathcal{N}_{l}\right]_{i, j} & := \begin{cases}K^{(i)} & \text { for } i=0, \ldots, l, j=0, \\
0 & \text { otherwise },\end{cases} \\
{\left[z_{l}\right]_{i}: } & =x^{(i)}, \quad i=0, \ldots, l, \\
{\left[g_{l}\right]_{i}: } & =f^{(i)}, \quad i=0, \ldots, l .
\end{aligned}
$$

Here, we use the convention that $\left(\begin{array}{l}i \\ j\end{array}\right)=0$ for $i<0, j<0$ or $j>i$.

For every $l \in \mathbb{N}_{0}$ and every $t \in \mathbb{I}$, we can now determine the local characteristic values of the triple $\left(\mathcal{M}_{l}(t), \mathcal{L}_{l}(t), \mathcal{N}_{l}(t)\right)$ by transforming it into the local condensed form given in [19]. These local quantities at a fixed point $\hat{t} \in \mathbb{I}$ are invariant under global equivalence transformations of the original triple $(M(t), C(t), K(t))$ of matrixvalued functions. To prove this, we use the following Lemmas.

LEMma 3.1. [11, Lemma 3.28] Let $D=A B C$ be the product of three sufficiently smooth matrix valued functions of appropriate dimensions. Then

$$
D^{(i)}=\sum_{j=0}^{i} \sum_{k=0}^{i-j}\left(\begin{array}{l}
i \\
j
\end{array}\right)\left(\begin{array}{c}
i-j \\
k
\end{array}\right) A^{(j)} B^{(k)} C^{(i-j-k)} .
$$

LEmma 3.2. For all integers $i, j, k, l$ with $i \geq 0, i \geq j \geq 0, i-j \geq k \geq 0$, we have

$$
\left(\begin{array}{l}
i \\
k
\end{array}\right)\left(\begin{array}{c}
i-k \\
l
\end{array}\right)\left(\begin{array}{c}
i-k-l \\
j
\end{array}\right)=\left(\begin{array}{c}
i \\
j
\end{array}\right)\left(\begin{array}{c}
i-j \\
k
\end{array}\right)\left(\begin{array}{c}
i-j-k \\
l
\end{array}\right)
$$




$$
\begin{aligned}
\left(\begin{array}{l}
i \\
k
\end{array}\right)\left(\begin{array}{c}
i-k \\
l
\end{array}\right)\left(\begin{array}{c}
i-k-l+2 \\
j+2
\end{array}\right) & =\left(\begin{array}{c}
i \\
j
\end{array}\right)\left(\begin{array}{c}
i-j \\
k
\end{array}\right)\left(\begin{array}{c}
i-j-k \\
l
\end{array}\right)+2\left(\begin{array}{c}
i \\
j+1
\end{array}\right)\left(\begin{array}{c}
i-j-1 \\
k
\end{array}\right)\left(\begin{array}{c}
i-j-k-1 \\
l
\end{array}\right) \\
& +\left(\begin{array}{c}
i \\
j+2
\end{array}\right)\left(\begin{array}{c}
i-j-2 \\
k
\end{array}\right)\left(\begin{array}{c}
i-j-k-2 \\
l
\end{array}\right) \\
\left(\begin{array}{c}
i \\
k
\end{array}\right)\left(\begin{array}{c}
i-k \\
l
\end{array}\right)\left(\begin{array}{c}
i-k-l+1 \\
j+2
\end{array}\right) & =\left(\begin{array}{c}
i \\
j+1
\end{array}\right)\left(\begin{array}{c}
i-j-1 \\
k
\end{array}\right)\left(\begin{array}{c}
i-j-1-k \\
l
\end{array}\right) \\
& +\left(\begin{array}{c}
i \\
j+2
\end{array}\right)\left(\begin{array}{c}
i-j-2 \\
k
\end{array}\right)\left(\begin{array}{c}
i-j-k-2 \\
l
\end{array}\right) \\
\left(\begin{array}{c}
i \\
k
\end{array}\right)\left(\begin{array}{c}
i-k \\
l
\end{array}\right)\left(\begin{array}{c}
i-k-l \\
j+2
\end{array}\right) & =\left(\begin{array}{c}
i \\
j+2
\end{array}\right)\left(\begin{array}{c}
i-j-2 \\
k
\end{array}\right)\left(\begin{array}{c}
i-j-k-2 \\
l
\end{array}\right) .
\end{aligned}
$$

Proof. The proof follows by straightforward calculations.

Now, we can show that the local quantities of the triple $\left(\mathcal{M}_{l}(\hat{t}), \mathcal{L}_{l}(\hat{t}), \mathcal{N}_{l}(\hat{t})\right)$ are invariant under global equivalence transformations of the original triple $(M(t), C(t)$, $K(t))$.

TheOrem 3.3. Consider two triples $(M, C, K)$ and $(\tilde{M}, \tilde{C}, \tilde{K})$ of sufficiently smooth matrix-valued functions that are globally equivalent via the transformation

$$
\tilde{M}=P M Q, \quad \tilde{C}=P C Q+2 P M \dot{Q}, \quad \tilde{K}=P K Q+P C \dot{Q}+P M \ddot{Q}
$$

according to Definition 2.1, with sufficiently smooth matrix-valued functions $P$ and Q. For $l \in \mathbb{N}_{0}$, let $\left(\mathcal{M}_{l}, \mathcal{L}_{l}, \mathcal{N}_{l}\right)$ and $\left(\tilde{\mathcal{M}}_{l}, \tilde{\mathcal{L}}_{l}, \tilde{\mathcal{N}}_{l}\right)$ be the corresponding inflated triples constructed as in (3.2) and introduce the block matrix functions

$$
\begin{aligned}
& {\left[\Pi_{l}\right]_{i, j}=\left(\begin{array}{l}
i \\
j
\end{array}\right) P^{(i-j)}, \quad\left[\Psi_{l}\right]_{i, j}= \begin{cases}\frac{i+2}{2} Q^{(i+1)} & \text { for } i=0, \ldots, l, j=0, \\
0 & \text { otherwise },\end{cases} } \\
& {\left[\Theta_{l}\right]_{i, j}=\left(\begin{array}{l}
i+2 \\
j+2
\end{array}\right) Q^{(i-j)}, \quad\left[\Sigma_{l}\right]_{i, j}= \begin{cases}Q^{(i+2)} & \text { for } i=0, \ldots, l, j=0, \\
0 & \text { otherwise. }\end{cases} }
\end{aligned}
$$

Then

$$
\left[\tilde{\mathcal{M}}_{l}(t), \tilde{\mathcal{L}}_{l}(t), \tilde{\mathcal{N}}_{l}(t)\right]=\Pi_{l}(t)\left[\mathcal{M}_{l}(t), \mathcal{L}_{l}(t), \mathcal{N}_{l}(t)\right]\left[\begin{array}{ccc}
\Theta_{l}(t) & 2 \Psi_{l}(t) & \Sigma_{l}(t) \\
0 & \Theta_{l}(t) & \Psi_{l}(t) \\
0 & 0 & \Theta_{l}(t)
\end{array}\right]
$$

for every $t \in \mathbb{I}$, and the corresponding matrix triples are locally equivalent.

Proof. First, we note that all matrix-valued functions $\mathcal{M}_{l}, \mathcal{L}_{l}, \mathcal{N}_{l}, \tilde{\mathcal{M}}_{l}, \tilde{\mathcal{L}}_{l}, \tilde{\mathcal{N}}_{l}, \Pi_{l}$, $\Psi_{l}, \Theta_{l}$ and $\Sigma_{l}$ are block lower triangular with the same block structure. Furthermore, $\mathcal{N}_{l}, \tilde{\mathcal{N}}_{l}, \mathcal{L}_{l}, \tilde{\mathcal{L}}_{l}, \Psi_{l}$ and $\Sigma_{l}$ have nonzero blocks only in the first block column. Using Lemma 3.1, we obtain

$$
\tilde{M}^{(i)}=\sum_{k_{1}=0}^{i} \sum_{k_{2}=0}^{i-k_{1}}\left(\begin{array}{c}
i \\
k_{1}
\end{array}\right)\left(\begin{array}{c}
i-k_{1} \\
k_{2}
\end{array}\right) P^{\left(k_{1}\right)} M^{\left(k_{2}\right)} Q^{\left(i-k_{1}-k_{2}\right)},
$$




$$
\begin{aligned}
& \tilde{C}^{(i)}=\sum_{k_{1}=0}^{i} \sum_{k_{2}=0}^{i-k_{1}}\left(\begin{array}{c}
i \\
k_{1}
\end{array}\right)\left(\begin{array}{c}
i-k_{1} \\
k_{2}
\end{array}\right)\left[P^{\left(k_{1}\right)} C^{\left(k_{2}\right)} Q^{\left(i-k_{1}-k_{2}\right)}+2 P^{\left(k_{1}\right)} M^{\left(k_{2}\right)} Q^{\left(i+1-k_{1}-k_{2}\right)}\right], \\
& \tilde{K}^{(i)}=\sum_{k_{1}=0}^{i} \sum_{k_{2}=0}^{i-k_{1}}\left(\begin{array}{c}
i \\
k_{1}
\end{array}\right)\left(\begin{array}{c}
i-k_{1} \\
k_{2}
\end{array}\right)\left[P^{\left(k_{1}\right)} K^{\left(k_{2}\right)} Q^{\left(i-k_{1}-k_{2}\right)}+P^{\left(k_{1}\right)} C^{\left(k_{2}\right)} Q^{\left(i+1-k_{1}-k_{2}\right)}\right. \\
& \left.+P^{\left(k_{1}\right)} M^{\left(k_{2}\right)} Q^{\left(i+2-k_{1}-k_{2}\right)}\right] .
\end{aligned}
$$

Inserting the definitions, shifting and inverting the summations and applying Lemma 3.2 lead to

$$
\begin{aligned}
& {\left[\Pi_{l} \mathcal{M}_{l} \Theta_{l}\right]_{i, j}=\sum_{l_{1}=j}^{i} \sum_{l_{2}=j}^{l_{1}}\left[\Pi_{l}\right]_{i, l_{1}}\left[\mathcal{M}_{l}\right]_{l_{1}, l_{2}}\left[\Theta_{l}\right]_{l_{2}, j}} \\
& =\sum_{l_{1}=j}^{i} \sum_{l_{2}=j}^{l_{1}}\left(\begin{array}{c}
i \\
l_{1}
\end{array}\right) P^{\left(i-l_{1}\right)}\left[\left(\begin{array}{l}
l_{1} \\
l_{2}
\end{array}\right) M^{\left(l_{1}-l_{2}\right)}+\left(\begin{array}{c}
l_{1} \\
l_{2}+1
\end{array}\right) C^{\left(l_{1}-l_{2}-1\right)}+\left(\begin{array}{c}
l_{1} \\
l_{2}+2
\end{array}\right) K^{\left(l_{1}-l_{2}-2\right)}\right]\left(\begin{array}{c}
l_{2}+2 \\
j+2
\end{array}\right) Q^{\left(l_{2}-j\right)} \\
& =\sum_{k_{1}=0}^{i-j} \sum_{l_{2}=j}^{k_{1}+j}\left(\begin{array}{c}
i \\
k_{1}+j
\end{array}\right) P^{\left(i-k_{1}-j\right)}\left[\left(\begin{array}{c}
k_{1}+j \\
l_{2}
\end{array}\right) M^{\left(k_{1}+j-l_{2}\right)}+\left(\begin{array}{c}
k_{1}+j \\
l_{2}+1
\end{array}\right) C^{\left(k_{1}+j-l_{2}-1\right)}\right. \\
& \left.+\left(\begin{array}{c}
k_{1}+j \\
l_{2}+2
\end{array}\right) K^{\left(k_{1}+j-l_{2}-2\right)}\right]\left(\begin{array}{c}
l_{2}+2 \\
j+2
\end{array}\right) Q^{\left(l_{2}-j\right)} \\
& =\left(\begin{array}{l}
i \\
j
\end{array}\right) \sum_{k_{1}=0}^{i-j} \sum_{k_{2}=0}^{i-j-k_{1}}\left(\begin{array}{c}
i-j \\
k_{1}
\end{array}\right)\left(\begin{array}{c}
i-j-k_{1} \\
k_{2}
\end{array}\right) P^{\left(k_{1}\right)} M^{\left(k_{2}\right)} Q^{\left(i-j-k_{1}-k_{2}\right)} \\
& +\left(\begin{array}{c}
i \\
j+1
\end{array}\right) \sum_{k_{1}=0}^{i-j-1} \sum_{k_{2}=0}^{i-j-1-k_{1}}\left(\begin{array}{c}
i-j-1 \\
k_{1}
\end{array}\right)\left(\begin{array}{c}
i-j-1-k_{1} \\
k_{2}
\end{array}\right) P^{\left(k_{1}\right)}\left[C^{\left(k_{2}\right)} Q^{\left(i-j-1-k_{1}-k_{2}\right)}\right. \\
& \left.+2 M^{\left(k_{2}\right)} Q^{\left(i-j-k_{1}-k_{2}\right)}\right] \\
& +\left(\begin{array}{c}
i \\
j+2
\end{array}\right) \sum_{k_{1}=0}^{i-j-2} \sum_{k_{2}=0}^{i-j-2-k_{1}}\left(\begin{array}{c}
i-j-2 \\
k_{1}
\end{array}\right)\left(\begin{array}{c}
i-j-2-k_{1} \\
k_{2}
\end{array}\right)\left[P^{\left(k_{1}\right)} K^{\left(k_{2}\right)} Q^{\left(i-j-2-k_{1}-k_{2}\right)}\right. \\
& \left.+P^{\left(k_{1}\right)} C^{\left(k_{2}\right)} Q^{\left(i-j-1-k_{1}-k_{2}\right)}+P^{\left(k_{1}\right)} M^{\left(k_{2}\right)} Q^{\left(i-j-k_{1}-k_{2}\right)}\right] \\
& =\left(\begin{array}{c}
i \\
j
\end{array}\right) \tilde{M}^{(i-j)}+\left(\begin{array}{c}
i \\
j+1
\end{array}\right) \tilde{C}^{(i-j-1)}+\left(\begin{array}{c}
i \\
j+2
\end{array}\right) \tilde{K}^{(i-j-2)}=\left[\tilde{\mathcal{M}}_{l}\right]_{i, j} .
\end{aligned}
$$

In the same way, we get

$$
\begin{aligned}
& {\left[\Pi_{l} \mathcal{L}_{l} \Theta_{l}\right]_{i, 0}+\left[2 \Pi_{l} \mathcal{M}_{l} \Psi_{l}\right]_{i, 0}=\sum_{l_{1}=0}^{i}\left[\Pi_{l}\right]_{i, l_{1}}\left[\mathcal{L}_{l}\right]_{l_{1}, 0}\left[\Theta_{l}\right]_{0,0}+2 \sum_{l_{1}=0}^{i} \sum_{l_{2}=0}^{l_{1}}\left[\Pi_{l}\right]_{i, l_{1}}\left[\mathcal{M}_{l}\right]_{l_{1}, l_{2}}\left[\Psi_{l}\right]_{l_{2}, 0} } \\
= & \sum_{l_{1}=0}^{i}\left(\begin{array}{c}
i \\
l_{1}
\end{array}\right) P^{\left(i-l_{1}\right)}\left[C^{\left(l_{1}\right)}+l_{1} K^{\left(l_{1}-1\right)}\right] Q+2 \sum_{l_{1}=0}^{i} \sum_{l_{2}=0}^{l_{1}}\left(\begin{array}{c}
i \\
l_{1}
\end{array}\right) P^{\left(i-l_{1}\right)}\left[\left(\begin{array}{l}
l_{1} \\
l_{2}
\end{array}\right) M^{\left(l_{1}-l_{2}\right)}\right.
\end{aligned}
$$




$$
\begin{aligned}
& \left.+\left(\begin{array}{c}
l_{1} \\
l_{2}+1
\end{array}\right) C^{\left(l_{1}-l_{2}-1\right)}+\left(\begin{array}{c}
l_{1} \\
l_{2}+2
\end{array}\right) K^{\left(l_{1}-l_{2}-2\right)}\right] \frac{l_{2}+2}{2} Q^{\left(l_{2}+1\right)} \\
= & \sum_{k_{1}=0}^{i} \sum_{k_{2}=0}^{i-k_{1}}\left(\begin{array}{c}
i \\
k_{1}
\end{array}\right)\left(\begin{array}{c}
i-k_{1} \\
k_{2}
\end{array}\right)\left[P^{\left(k_{1}\right)} C^{\left(k_{2}\right)} Q^{\left(i-k_{1}-k_{2}\right)}+2 P^{\left(k_{1}\right)} M^{\left(k_{2}\right)} Q^{\left(i+1-k_{1}-k_{2}\right)}\right] \\
+ & i \sum_{k_{1}=0}^{i-1} \sum_{k_{2}=0}^{i-1-k_{1}}\left(\begin{array}{c}
i-1 \\
k_{1}
\end{array}\right)\left(\begin{array}{c}
i-1-k_{1} \\
k_{2}
\end{array}\right)\left[P^{\left(k_{1}\right)} K^{\left(k_{2}\right)} Q^{\left(i-1-k_{1}-k_{2}\right)}\right. \\
= & \tilde{C}^{(i)}+i \tilde{K}^{(i-1)}=\left[\tilde{\mathcal{L}}_{l}\right]_{i, 0},
\end{aligned}
$$

and

$$
\begin{aligned}
& {\left[\Pi_{l} \mathcal{N}_{l} \Theta_{l}\right]_{i, 0}+\left[\Pi_{l} \mathcal{L}_{l} \Psi_{l}\right]_{i, 0}+\left[\Pi_{l} \mathcal{M}_{l} \Sigma_{l}\right]_{i, 0}=} \\
= & \sum_{l_{1}=0}^{i}\left[\Pi_{l}\right]_{i, l_{1}}\left[\mathcal{N}_{l}\right]_{l_{1}, 0}\left[\Theta_{l}\right]_{0,0}+\sum_{l_{1}=0}^{i}\left[\Pi_{l}\right]_{i, l_{1}}\left[\mathcal{L}_{l}\right]_{l_{1}, 0}\left[\Psi_{l}\right]_{0,0}+\sum_{l_{1}=0}^{i} \sum_{l_{2}=0}^{l_{1}}\left[\Pi_{l}\right]_{i, l_{1}}\left[\mathcal{M}_{l}\right]_{l_{1}, l_{2}}\left[\Sigma_{l}\right]_{l_{2}, 0} \\
= & \sum_{l_{1}=0}^{i}\left(\begin{array}{c}
i \\
l_{1}
\end{array}\right) P^{\left(i-l_{1}\right)} K^{\left(l_{1}\right)} Q+\sum_{l_{1}=0}^{i}\left(\begin{array}{c}
i \\
l_{1}
\end{array}\right) P^{\left(i-l_{1}\right)}\left[C^{\left(l_{1}\right)}+l_{1} K^{\left(l_{1}-1\right)}\right] Q^{(1)} \\
+ & \sum_{l_{1}=0}^{i} \sum_{l_{2}=0}^{l_{1}}\left(\begin{array}{c}
i \\
l_{1}
\end{array}\right) P^{\left(i-l_{1}\right)}\left[\left(\begin{array}{c}
l_{1} \\
l_{2}
\end{array}\right) M^{\left(l_{1}-l_{2}\right)}+\left(\begin{array}{c}
l_{1} \\
l_{2}+1
\end{array}\right) C^{\left(l_{1}-l_{2}-1\right)}+\left(\begin{array}{c}
l_{1} \\
l_{2}+2
\end{array}\right) K^{\left(l_{1}-l_{2}-2\right)}\right] Q^{\left(l_{2}+2\right)} \\
= & \sum_{k_{1}=0}^{i} \sum_{k_{2}=0}^{i-k_{1}}\left(\begin{array}{c}
i \\
k_{1}
\end{array}\right)\left(\begin{array}{c}
i-k_{1} \\
k_{2}
\end{array}\right)\left[P^{\left(k_{1}\right)}\left(K^{\left(k_{2}\right)} Q^{\left(i-k_{1}-k_{2}\right)}+C^{\left(k_{2}\right)} Q^{\left(i+1-k_{1}-k_{2}\right)}+M^{\left(k_{2}\right)} Q^{\left(i+2-k_{1}-k_{2}\right)}\right)\right] \\
= & \tilde{K}^{(i)}=\left[\tilde{\mathcal{N}}_{l}\right]_{i, 0} .
\end{aligned}
$$

As a consequence of Theorem 3.3, the local characteristic values $\left(\tilde{r}_{l}, \tilde{d}_{l}^{(1)}, \tilde{a}_{l}\right.$, $\left.\tilde{s}_{l}^{(M C K)}, \tilde{s}_{l}^{(M K)}, \tilde{s}_{l}^{(C K)}, \tilde{s}_{l}^{(M C)}, \tilde{u}_{l}, \tilde{v}_{l}\right)$ of the inflated triple $\left(\mathcal{M}_{l}(\hat{t}), \mathcal{L}_{l}(\hat{t}), \mathcal{N}_{l}(\hat{t})\right)$ at a fixed point $\hat{t}$ are well-defined for equivalent triples of matrix-valued functions and for each $l \in \mathbb{N}_{0}$. These quantities are numerically computable via a number of numerical rank decisions. Next, we show how these local quantities of the inflated triple $\left(\mathcal{M}_{l}(\hat{t}), \mathcal{L}_{l}(\hat{t}), \mathcal{N}_{l}(\hat{t})\right)$ are related to the global characteristic values of the original triple $(M, C, K)$ at the point $\hat{t}$. For convenience of presentation, we restrict ourselves to the case that $\mu \leq 2$ in the following.

Theorem 3.4. Consider a triple $(M, C, K)$ of sufficiently smooth matrix-valued functions $M, C, K \in C\left(\mathbb{I}, \mathbb{C}^{m, n}\right)$ with well-defined strangeness index $\mu \leq 2$ and sequence of global characteristic values $\left(r_{i}, d_{i}^{(1)}, a_{i}, s_{i}^{(M C K)}, s_{i}^{(C K)}, s_{i}^{(M C)}, s_{i}^{(M K)}, u_{i}, v_{i}\right)$, $i \in \mathbb{N}_{0}$. Furthermore, let $\left(\mathcal{M}_{l}(\hat{t}), \mathcal{L}_{l}(\hat{t}), \mathcal{N}_{l}(\hat{t})\right)$ be the corresponding inflated matrix triple at a fixed $\hat{t} \in \mathbb{I}$ with local characteristic values $\left(\tilde{r}_{l}, \tilde{d}_{l}^{(1)}, \tilde{a}_{l}, \tilde{s}_{l}^{(M C K)}, \tilde{s}_{l}^{(C K)}, \tilde{s}_{l}^{(M C)}\right.$, 
$\left.\tilde{s}_{l}^{(M K)}, \tilde{u}_{l}, \tilde{v}_{l}\right)$. Then, for $l=0,1,2$, we have

$$
\begin{aligned}
& \operatorname{rank}\left[\mathcal{M}_{l}, \mathcal{L}_{l}, \mathcal{N}_{l}\right]=(l+1) m-\sum_{i=0}^{l} v_{i}, \\
& \operatorname{rank}\left[\mathcal{M}_{l}, \mathcal{L}_{l}\right]=(l+1) m-\sum_{i=0}^{l} c_{i}-\sum_{i=0}^{l} v_{i}, \\
& \operatorname{rank}\left[\mathcal{M}_{l}\right]=\tilde{r}_{l}=(l+1) m-\sum_{i=0}^{l} q_{i}-\sum_{i=0}^{l} c_{i}-\sum_{i=0}^{l} v_{i},
\end{aligned}
$$

using the definitions as in Lemma 2.6, and

$$
\begin{aligned}
& \tilde{d}_{l}^{(1)}=k_{l}-p_{l}+b_{l}, \\
& \tilde{a}_{l}=b_{l}=c_{l}-s_{l}^{(M C K)}-s_{l}^{(C K)}-s_{l}^{(M K)}+s_{l-1}, \\
& \tilde{s}_{l}^{(M C K)}=\sum_{i=0}^{l} c_{i}-b_{l-1}-p_{l}-\sum_{i=1}^{l}\left(d_{i}-t_{i}\right), \\
& \tilde{s}_{l}^{(C K)}=b_{l-1}-b_{l}+p_{l}, \\
& \tilde{s}_{l}^{(M C)}=\sum_{i=0}^{l} q_{i}-\sum_{i=0}^{l} c_{i}+\sum_{i=1}^{l}\left(d_{i}-t_{i}\right)+p_{l}-k_{l}, \\
& \tilde{s}_{l}^{(M K)}=\sum_{i=1}^{l}\left(d_{i}-t_{i}\right), \\
& \tilde{v}_{l}=\sum_{i=0}^{l} v_{i}, \\
& \tilde{u}_{l}=(l+1) u_{0}+(l+1) a_{0}+l k_{0}-\sum_{i=0}^{l} k_{i}-\sum_{i=0}^{l} b_{i} .
\end{aligned}
$$

Proof. We omit the proof for ease of presentation. The proof is given in $[18,19]$.

From the relations in (3.4) we can determine recursive formulas for the global characteristic values of the original matrix triple $(M, C, K)$.

Corollary 3.5. Let the strangeness index $\mu$ of the matrix triple $(M, C, K)$ be well-defined with $\mu \leq 2$ and let $\left(\tilde{r}_{l}, \tilde{d}_{l}^{(1)}, \tilde{a}_{l}, \tilde{s}_{l}^{(M C K)}, \tilde{s}_{l}^{(C K)}, \tilde{s}_{l}^{(M C)}, \tilde{s}_{l}^{(M K)}, \tilde{u}_{l}, \tilde{v}_{l}\right)$, $l=0, \ldots, \mu$ be the sequence of the local characteristic values of $\left(\mathcal{M}_{l}, \mathcal{L}_{l}, \mathcal{N}_{l}\right)$ for some $t \in \mathbb{I}$. Then, for the sequence $\left(r_{i}, d_{i}^{(1)}, a_{i}, s_{i}^{(M C K)}, s_{i}^{(C K)}, s_{i}^{(M C)}, s_{i}^{(M K)}, u_{i}, v_{i}\right)$ of the global characteristic values of $(M, C, K)$, it holds that

$$
c_{0}=\tilde{a}_{0}+\tilde{s}_{0}^{(M C K)}+\tilde{s}_{0}^{(C K)}+\tilde{s}_{0}^{(M K)},
$$




$$
\begin{aligned}
& c_{i+1}=\left(\tilde{a}_{i+1}-\tilde{a}_{i}\right)+\left(\tilde{s}_{i+1}^{(M C K)}-\tilde{s}_{i}^{(M C K)}\right)+\left(\tilde{s}_{i+1}^{(C K)}-\tilde{s}_{i}^{(C K)}\right)+\left(\tilde{s}_{i+1}^{(M K)}-\tilde{s}_{i}^{(M K)}\right), \\
& q_{0}=\tilde{d}_{0}^{(1)}+\tilde{s}_{0}^{(M C K)}+\tilde{s}_{0}^{(C K)}+\tilde{s}_{0}^{(M C)} \\
& q_{i+1}=\left(\tilde{d}_{i+1}^{(1)}-\tilde{d}_{i}^{(1)}\right)+\left(\tilde{s}_{i+1}^{(M C K)}-\tilde{s}_{i}^{(M C K)}\right)+\left(\tilde{s}_{i+1}^{(C K)}-\tilde{s}_{i}^{(C K)}\right)+\left(\tilde{s}_{i+1}^{(M C)}-\tilde{s}_{i}^{(M C)}\right), \\
& v_{0}=m-c_{0}-q_{0}-\tilde{r}_{0}, \\
& v_{i+1}=m-c_{i+1}-q_{i+1}-\left(\tilde{r}_{i+1}-\tilde{r}_{i}\right) \\
& s_{i}^{(M C K)}+s_{i}^{(C K)}+s_{i}=c_{i}-\tilde{a}_{i}, \\
& s_{i}^{(M C K)}+s_{i}^{(M C)}+s_{i-1}=q_{i}-\tilde{d}_{i}^{(1)}-\tilde{s}_{i}^{(C K)} .
\end{aligned}
$$

Proof. The relations follow directly from Theorem 3.4 and from the definitions in Lemma 2.6, since

$$
\begin{aligned}
& \tilde{a}_{i+1}-\tilde{a}_{i}=b_{i+1}-b_{i}, \\
& \tilde{s}_{i+1}^{(M C K)}-\tilde{s}_{i}^{(M C K)}+\tilde{s}_{i+1}^{(C K)}-\tilde{s}_{i}^{(C K)}=c_{i+1}-d_{i+1}+t_{i+1}-b_{i+1}+b_{i}, \\
& \tilde{s}_{i+1}^{(M K)}-\tilde{s}_{i}^{(M K)}=d_{i+1}-t_{i+1}, \\
& \tilde{d}_{i+1}^{(1)}-\tilde{d}_{i}^{(1)}=k_{i+1}-k_{i}+p_{i}-p_{i+1}+b_{i+1}-b_{i}, \\
& \tilde{s}_{i+1}^{(M C)}-\tilde{s}_{i}^{(M C)}=q_{i+1}-c_{i+1}+d_{i+1}-t_{i+1}+p_{i+1}-p_{i}+k_{i}-k_{i+1}, \\
& m-c_{i+1}-q_{i+1}-\left(\tilde{r}_{i+1}-\tilde{r}_{i}\right)=m-c_{i+1}-q_{i+1}-m+q_{i+1}+c_{i+1}+v_{i+1}=v_{i+1}, \\
& c_{i}-\tilde{a}_{i}=c_{i}-c_{i}+s_{i}^{(M C K)}+s_{i}^{(C K)}+s_{i}^{(M K)}-s_{i-1}=s_{i}^{(M C K)}+s_{i}^{(C K)}+s_{i}, \\
& q_{i}-\tilde{d}_{i}^{(1)}-\tilde{s}_{i}^{(C K)}=q_{i}-k_{i}-b_{i-1}=s_{i}^{(M C K)}+s_{i}^{(M C)}+s_{i-1} \cdot
\end{aligned}
$$

Thus, the recursive formulas given in Corollary 3.5 enable the determination of the strangeness index $\mu$ in a numerically computable way by determining the local characteristic values of the inflated triple $\left(\mathcal{M}_{l}, \mathcal{L}_{l}, \mathcal{N}_{l}\right)$ for each time $t \in \mathbb{I}$. The differential-algebraic system in the $i$-th reduction step is strangeness-free if the sums $s_{i}^{(M C K)}+s_{i}^{(M C)}+s_{i-1}$ and $s_{i}^{(M C K)}+s_{i}^{(C K)}+s_{i}$ vanish, since then $s_{i}^{(M C K)}=s_{i}^{(C K)}=$ $s_{i}^{(M K)}=s_{i}^{(M C)}=0$, as all summands are nonnegative integer values. Finally, for the characteristic values of the strangeness-free system, we get

$$
\begin{aligned}
a_{\mu} & =\sum_{i=0}^{\mu} c_{i}=\operatorname{rank}\left[\mathcal{M}_{\mu}, \mathcal{L}_{\mu}, \mathcal{N}_{\mu}\right]-\operatorname{rank}\left[\mathcal{M}_{\mu}, \mathcal{L}_{\mu}\right], \\
d_{\mu}^{(1)} & =\sum_{i=0}^{\mu} q_{i}-\sum_{i=0}^{\mu-1} c_{i} \\
& =\operatorname{rank}\left[\mathcal{M}_{\mu}, \mathcal{L}_{\mu}\right]-\tilde{r}_{\mu}+\operatorname{rank}\left[\mathcal{M}_{\mu-1}, \mathcal{L}_{\mu-1}\right]-\operatorname{rank}\left[\mathcal{M}_{\mu-1}, \mathcal{L}_{\mu-1}, \mathcal{N}_{\mu-1}\right], \\
v_{\mu} & =\tilde{v}_{\mu}-\tilde{v}_{\mu-1}, \\
d_{\mu}^{(2)} & =m-a_{\mu}-d_{\mu}^{(1)}-v_{\mu} .
\end{aligned}
$$


Further, we can extract a strangeness-free triple $(\hat{M}, \hat{C}, \hat{K})$ from the inflated system with characteristic values $\hat{r}=d_{\mu}^{(2)}, \hat{d}^{(1)}=d_{\mu}^{(1)}, \hat{a}=a_{\mu}, \hat{u}=u_{\mu}, \hat{v}=v_{\mu}$ and $\hat{s}^{(M C K)}=$ $\hat{s}^{(C K)}=\hat{s}^{(M K)}=\hat{s}^{(M C)}=0$ using only local information from $\left(\mathcal{M}_{\mu}(t), \mathcal{L}_{\mu}(t), \mathcal{N}_{\mu}(t)\right)$.

THEOREM 3.6. Consider a linear second order differential-algebraic system (1.1) with well-defined strangeness index $\mu \leq 2$. Then the inflated triple $\left(\mathcal{M}_{\mu}, \mathcal{L}_{\mu}, \mathcal{N}_{\mu}\right)$ associated with $(M, C, K)$ has the following properties:

1. For all $t \in \mathbb{I}$, it holds that

$$
\operatorname{rank} \mathcal{M}_{\mu}(t)=(\mu+1) m-a_{\mu}-\tilde{v}_{\mu}-d_{\mu}^{(1)}-\sum_{i=0}^{\mu-1} c_{i},
$$

such that there exists a smooth matrix function $Z$ with orthonormal columns and size $\left((\mu+1) m, a_{\mu}+\tilde{v}_{\mu}+d_{\mu}^{(1)}+\sum_{i=0}^{\mu-1} c_{i}\right)$ satisfying

$$
Z^{H} \mathcal{M}_{\mu}=0
$$

2. For all $t \in \mathbb{I}$, we have

$$
\begin{aligned}
& \operatorname{rank}\left[\mathcal{M}_{\mu}(t), \mathcal{L}_{\mu}(t)\right]=(\mu+1) m-a_{\mu}-\tilde{v}_{\mu}, \\
& \operatorname{rank}\left[\mathcal{M}_{\mu}(t), \mathcal{L}_{\mu}(t), \mathcal{N}_{\mu}(t)\right]=(\mu+1) m-\tilde{v}_{\mu},
\end{aligned}
$$

such that without loss of generality, $Z$ can be partitioned into $Z=\left[Z_{2}, Z_{3}, Z_{4}\right]$ with $Z_{2}$ of size $\left((\mu+1) m, d_{\mu}^{(1)}+\sum_{i=0}^{\mu-1} c_{i}\right), Z_{3}$ of size $\left((\mu+1) m, a_{\mu}\right)$ and $Z_{4}$ of size $\left((\mu+1) m, \tilde{v}_{\mu}\right)$ such that

$$
Z_{3}^{H} \mathcal{L}_{\mu}=0, \quad Z_{4}^{H} \mathcal{L}_{\mu}=0, \quad Z_{4}^{H} \mathcal{N}_{\mu}=0
$$

3. For all $t \in \mathbb{I}$, we have

$$
\begin{aligned}
& \operatorname{rank}\left(Z_{3}^{H} \mathcal{N}_{\mu}\left[\begin{array}{llll}
I_{n} & 0 & \ldots & 0
\end{array}\right]^{H}\right)=a_{\mu}, \\
& \operatorname{rank}\left(Z_{2}^{H} \mathcal{L}_{\mu}\left[\begin{array}{llll}
I_{n} & 0 & \ldots & 0
\end{array}\right]^{H}\right)=d_{\mu}^{(1)}+\sum_{i=0}^{\mu-1} c_{i},
\end{aligned}
$$

such that there exists a smooth matrix function $T_{3}$ with orthonormal columns and size $\left(n, n-a_{\mu}\right)$, with $n-a_{\mu}=d_{\mu}^{(2)}+d_{\mu}^{(1)}+u_{\mu}$ satisfying

$$
Z_{3}^{H} \mathcal{N}_{\mu}\left[\begin{array}{llll}
I_{n} & 0 & \cdots & 0
\end{array}\right]^{H} T_{3}=0 .
$$

4. For all $t \in \mathbb{I}$, we have

$$
\operatorname{rank}\left(Z_{2}^{H} \mathcal{L}_{\mu}\left[\begin{array}{llll}
I_{n} & 0 & \ldots & 0
\end{array}\right]^{H} T_{3}\right)=d_{\mu}^{(1)},
$$


such that there exists a smooth matrix function $Z_{1}$ of size $\left(d_{\mu}^{(1)}+\sum_{i=0}^{\mu-1} c_{i}, d_{\mu}^{(1)}\right)$ with orthonormal columns such that

$$
\operatorname{rank}\left(Z_{1}^{H} Z_{2}^{H} \mathcal{L}_{\mu}\left[\begin{array}{cccc}
I_{n} & 0 & \cdots & 0
\end{array}\right]^{H}\right)=d_{\mu}^{(1)} .
$$

Furthermore, there exists a smooth matrix function $T_{2}$ of size $\left(n-a_{\mu}, n-\right.$ $\left.a_{\mu}-d_{\mu}^{(1)}\right)$ with orthonormal columns, such that

$$
Z_{1}^{H} Z_{2}^{H} \mathcal{L}_{\mu}\left[\begin{array}{cccc}
I_{n} & 0 & \cdots & 0
\end{array}\right]^{H} T_{3} T_{2}=0 .
$$

5. For all $t \in \mathbb{I}$, it holds that $\operatorname{rank}\left(M T_{3} T_{2}\right)=d_{\mu}^{(2)}$. This implies the existence of a smooth matrix function $Z_{0}$ with orthonormal columns and size $\left(m, d_{\mu}^{(2)}\right)$ such that $Z_{0}^{H} M$ has constant rank $d_{\mu}^{(2)}$.

Proof. The proof is given in Appendix.

From the results of Theorem 3.6 we can construct a triple of matrix-valued functions

$$
(\hat{M}, \hat{C}, \hat{K})=\left(\left[\begin{array}{c}
\hat{M}_{1} \\
0 \\
0 \\
0
\end{array}\right],\left[\begin{array}{c}
\hat{C}_{1} \\
\hat{C}_{2} \\
0 \\
0
\end{array}\right],\left[\begin{array}{c}
\hat{K}_{1} \\
\hat{K}_{2} \\
\hat{K}_{3} \\
0
\end{array}\right]\right)
$$

with entries

$$
\begin{aligned}
& \hat{M}_{1}=Z_{0}^{H} M, \quad \hat{C}_{1}=Z_{0}^{H} C, \quad \hat{K}_{1}=Z_{0}^{H} K, \quad \hat{C}_{2}=Z_{1}^{H} Z_{2}^{H} \mathcal{L}_{\mu}\left[\begin{array}{cccc}
I_{n} & 0 & \cdots & 0
\end{array}\right]^{H}, \\
& \hat{K}_{2}=Z_{1}^{H} Z_{2}^{H} \mathcal{N}_{\mu}\left[\begin{array}{llll}
I_{n} & 0 & \cdots & 0
\end{array}\right]^{H}, \quad \hat{K}_{3}=Z_{3}^{H} \mathcal{N}_{\mu}\left[\begin{array}{llll}
I_{n} & 0 & \cdots & 0
\end{array}\right]^{H},
\end{aligned}
$$

which has the same size as the original triple $(M, C, K)$. We can show that this triple is strangeness-free with the same characteristic values as the strangeness-free system (2.4).

THEOREM 3.7. Let the strangeness index $\mu$ of $(M, C, K)$ be well-defined with $\mu \leq 2$ and global characteristic values $\left(r_{i}, d_{i}^{(1)}, a_{i}, s_{i}^{(M C K)}, s_{i}^{(M C)}, s_{i}^{(M K)}, s_{i}^{(C K)}, u_{i}, v_{i}\right)$, for $i=0, \ldots, \mu$. Then, the triple $(\hat{M}, \hat{C}, \hat{K})$, constructed as in (3.6), has a well-defined strangeness index $\hat{\mu}=0$ and the global characteristic values of $(\hat{M}(t), \hat{C}(t), \hat{K}(t))$ are given by

$$
\left(\hat{r}, \hat{d}^{(1)}, \hat{a}, \hat{s}^{(M C K)}, \hat{s}^{(M C)}, \hat{s}^{(M K)}, \hat{s}^{(C K)}, \hat{v}\right)=\left(d_{\mu}^{(2)}, d_{\mu}^{(1)}, a_{\mu}, 0,0,0,0, v_{\mu}\right)
$$

uniformly for all $t \in \mathbb{I}$.

Proof. In the following, we omit the argument $t$ for simplicity. By construction the columns of $T_{3}$ defined in Theorem 3.6 form a basis of kernel $\hat{K}_{3}$ and the columns of 
$T_{2}$ form a basis of kernel $\left(\hat{C}_{2} T_{3}\right)$. We consider the matrix $T=T_{3} T_{2}$. Because $\hat{M}_{1}$ has full row rank, without loss of generality, we can split $T$ into $T=\left[\begin{array}{cc}T_{1}^{\prime} & T_{4}^{\prime}\end{array}\right]$ in such a way that $\hat{M}_{1} T_{1}^{\prime}$ is nonsingular. By choosing $T_{3}^{\prime}$ such that $\hat{K}_{3} T_{3}^{\prime}$ is also nonsingular and $T_{2}^{\prime}$ such that $\hat{C}_{2} T_{2}^{\prime}$ is nonsingular and $\hat{K}_{3} T_{2}^{\prime}=0$, we get a nonsingular matrix $\hat{T}=\left[\begin{array}{llll}T_{1}^{\prime} & T_{2}^{\prime} & T_{3}^{\prime} & T_{4}^{\prime}\end{array}\right]$. By multiplication with this matrix from the right we get the following local equivalence

$$
\begin{aligned}
& (\hat{M}, \hat{C}, \hat{K})=\left(\left[\begin{array}{c}
\hat{M}_{1} \\
0 \\
0 \\
0
\end{array}\right],\left[\begin{array}{c}
\hat{C}_{1} \\
\hat{C}_{2} \\
0 \\
0
\end{array}\right],\left[\begin{array}{c}
\hat{K}_{1} \\
\hat{K}_{2} \\
\hat{K}_{3} \\
0
\end{array}\right]\right) \\
& \sim\left(\left[\begin{array}{cccc}
\hat{M}_{1} T_{1}^{\prime} & \hat{M}_{1} T_{2}^{\prime} & \hat{M}_{1} T_{3}^{\prime} & \hat{M}_{1} T_{4}^{\prime} \\
0 & 0 & 0 & 0 \\
0 & 0 & 0 & 0 \\
0 & 0 & 0 & 0
\end{array}\right],\left[\begin{array}{cccc}
\hat{C}_{1} T_{1}^{\prime} & \hat{C}_{1} T_{2}^{\prime} & \hat{C}_{1} T_{3}^{\prime} & \hat{C}_{1} T_{4}^{\prime} \\
\hat{C}_{2} T_{1}^{\prime} & \hat{C}_{2} T_{2}^{\prime} & \hat{C}_{2} T_{3}^{\prime} & \hat{C}_{2} T_{4}^{\prime} \\
0 & 0 & 0 & 0 \\
0 & 0 & 0 & 0
\end{array}\right]\right. \\
& \left.\left[\begin{array}{cccc}
\hat{K}_{1} T_{1}^{\prime} & \hat{K}_{1} T_{2}^{\prime} & \hat{K}_{1} T_{3}^{\prime} & \hat{K}_{1} T_{4}^{\prime} \\
\hat{K}_{2} T_{1}^{\prime} & \hat{K}_{2} T_{2}^{\prime} & \hat{K}_{2} T_{3}^{\prime} & \hat{K}_{2} T_{4}^{\prime} \\
\hat{K}_{3} T_{1}^{\prime} & \hat{K}_{3} T_{2}^{\prime} & \hat{K}_{3} T_{3}^{\prime} & \hat{K}_{3} T_{4}^{\prime} \\
0 & 0 & 0 & 0
\end{array}\right]\right) \\
& \sim\left(\left[\begin{array}{cccc}
\hat{M}_{1} T_{1}^{\prime} & \hat{M}_{1} T_{2}^{\prime} & \hat{M}_{1} T_{3}^{\prime} & \hat{M}_{1} T_{4}^{\prime} \\
0 & 0 & 0 & 0 \\
0 & 0 & 0 & 0 \\
0 & 0 & 0 & 0
\end{array}\right],\left[\begin{array}{cccc}
\hat{C}_{1} T_{1}^{\prime} & \hat{C}_{1} T_{2}^{\prime} & \hat{C}_{1} T_{3}^{\prime} & \hat{C}_{1} T_{4}^{\prime} \\
0 & \hat{C}_{2} T_{2}^{\prime} & 0 & 0 \\
0 & 0 & 0 & 0 \\
0 & 0 & 0 & 0
\end{array}\right],\right. \\
& \left.\left[\begin{array}{cccc}
\hat{K}_{1} T_{1}^{\prime} & \hat{K}_{1} T_{2}^{\prime} & \hat{K}_{1} T_{3}^{\prime} & \hat{K}_{1} T_{4}^{\prime} \\
\hat{K}_{2} T_{1}^{\prime} & \hat{K}_{2} T_{2}^{\prime} & \hat{K}_{2} T_{3}^{\prime} & \hat{K}_{2} T_{4}^{\prime} \\
0 & 0 & \hat{K}_{3} T_{3}^{\prime} & 0 \\
0 & 0 & 0 & 0
\end{array}\right]\right) \\
& \sim\left(\left[\begin{array}{cccc}
\hat{M}_{1} T_{1}^{\prime} & 0 & 0 & 0 \\
0 & 0 & 0 & 0 \\
0 & 0 & 0 & 0 \\
0 & 0 & 0 & 0
\end{array}\right],\left[\begin{array}{cccc}
\star & \star & \star & \star \\
0 & \hat{C}_{2} T_{2}^{\prime} & 0 & 0 \\
0 & 0 & 0 & 0 \\
0 & 0 & 0 & 0
\end{array}\right],\left[\begin{array}{cccc}
\star & \star & \star & \star \\
\star & \star & \star & \star \\
0 & 0 & \hat{K}_{3} T_{3}^{\prime} & 0 \\
0 & 0 & 0 & 0
\end{array}\right]\right) \\
& \sim\left(\left[\begin{array}{cccc}
I_{d_{\mu}^{(2)}} & 0 & 0 & 0 \\
0 & 0 & 0 & 0 \\
0 & 0 & 0 & 0 \\
0 & 0 & 0 & 0
\end{array}\right],\left[\begin{array}{cccc}
\star & \star & \star & \star \\
0 & I_{d_{\mu}^{(1)}} & 0 & 0 \\
0 & 0 & 0 & 0 \\
0 & 0 & 0 & 0
\end{array}\right],\left[\begin{array}{cccc}
\star & \star & \star & \star \\
\star & \star & \star & \star \\
0 & 0 & I_{a_{\mu}} & 0 \\
0 & 0 & 0 & 0
\end{array}\right]\right) \text {. }
\end{aligned}
$$

From the last triple we obtain $\hat{r}=d_{\mu}^{(2)}, \hat{d}^{(1)}=d_{\mu}^{(1)}, \hat{a}=a_{\mu}, \hat{s}^{(M C K)}=\hat{s}^{(M C)}=$ $\hat{s}^{(M K)}=\hat{s}^{(C K)}=0$ and $\hat{v}=v_{\mu}$ from Lemma 2.3 .

Thus, we have derived an index reduction method that allows us to extract 
a strangeness-free triple from the original triple of matrix-valued functions and its derivatives. The matrix-valued functions $Z_{0}, Z_{1}, Z_{2}$ and $Z_{3}$ as given in Theorem 3.6 can be determined via numerical rank decisions, e.g., using a singular value decomposition or a rank revealing QR decomposition; see [7]. Setting the inhomogeneities $\hat{f}_{1}=Z_{0}^{H} f, \hat{f}_{2}=Z_{1}^{H} Z_{2}^{H} g_{\mu}, \hat{f}_{3}=Z_{3}^{H} g_{\mu}$ and $\hat{f}_{4}=0$ accordingly (assuming that the system is solvable) we obtain a differential-algebraic system

$$
\hat{M}(t) \ddot{x}+\hat{C}(t) \dot{x}+\hat{K}(t) x=\hat{f}(t)
$$

from the inflated differential-algebraic equation (3.1). Setting $\hat{f}_{4}=0$ in (3.7) can be seen as a regularization, since an unsolvable problem is replaced by a solvable one. System (3.7) is strangeness-free and has the same size and also the same solution set as the original system (1.1), since only transformations from the left are involved. In the following we give an example to illustrate the index reduction procedure.

EXAMPLE 3.8. We consider again the linear second order system (1.3) of strangeness index $\mu=2$ with characteristic values $d_{\mu}^{(2)}=1, d_{\mu}^{(1)}=0, a_{\mu}=2, v_{\mu}=0$ and $u_{\mu}=0$. The matrix triple corresponding to the extended system (3.1) is given by

$$
\mathcal{M}_{2}(t)=\left[\begin{array}{ccc|ccc|ccc}
t & 0 & 0 & 0 & 0 & 0 & 0 & 0 & 0 \\
0 & 1 & 1 & 0 & 0 & 0 & 0 & 0 & 0 \\
0 & t & t & 0 & 0 & 0 & 0 & 0 & 0 \\
\hline 2 & 0 & 0 & t & 0 & 0 & 0 & 0 & 0 \\
0 & 0 & 0 & 0 & 1 & 1 & 0 & 0 & 0 \\
0 & 1 & 1 & 0 & t & t & 0 & 0 & 0 \\
\hline 1 & 0 & 0 & 3 & 0 & 0 & t & 0 & 0 \\
0 & 1 & 0 & 0 & 0 & 0 & 0 & 1 & 1 \\
0 & 1+t & 1 & 0 & 2 & 2 & 0 & t & t
\end{array}\right],
$$

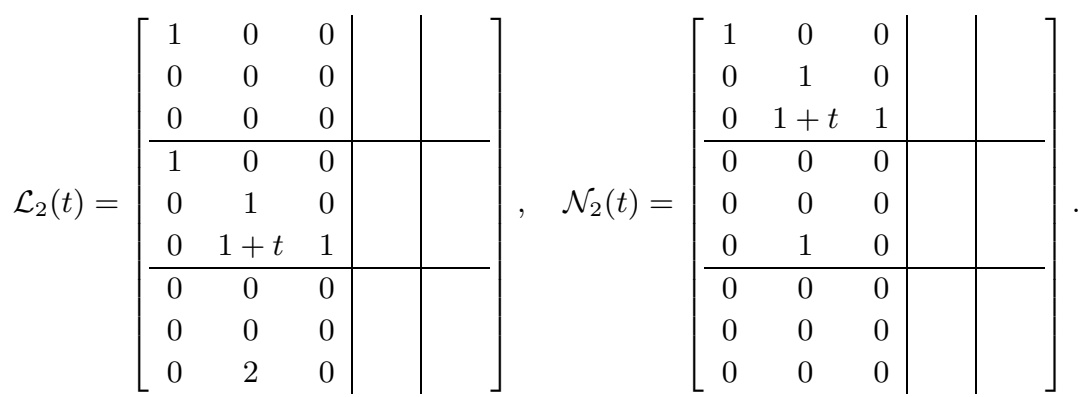

We have

$$
\begin{aligned}
\operatorname{rank}\left[\mathcal{M}_{2}(t), \mathcal{L}_{2}(t), \mathcal{N}_{2}(t)\right] & =9=(\mu+1) m-\tilde{v}_{\mu} \\
\operatorname{rank}\left[\mathcal{M}_{2}(t), \mathcal{L}_{2}(t)\right] & =7=(\mu+1) m-a_{\mu}-\tilde{v}_{\mu} \\
\operatorname{rank}\left[\mathcal{M}_{2}(t)\right] & =6=(\mu+1) m-d_{\mu}^{(1)}-c_{0}-c_{1}-a_{\mu}-\tilde{v}_{\mu}
\end{aligned}
$$


independent of $t \in \mathbb{I}$ and we can choose

$$
\begin{aligned}
Z_{3}^{H} & =\left[\begin{array}{ccccccccc}
0 & -1 & 0 & 0 & -2 & 0 & 0 & -t & 1 \\
0 & -t & 1 & 0 & 0 & 0 & 0 & 0 & 0
\end{array}\right], \\
Z_{2}^{H} & =\left[\begin{array}{lllllllll}
0 & -1 & 0 & 0 & -t & 1 & 0 & 0 & 0
\end{array}\right], \\
T_{3} & =\left[\begin{array}{lll}
1 & 0 & 0
\end{array}\right]^{H}, \quad T_{2}=1 .
\end{aligned}
$$

Then we have

$$
\begin{aligned}
& \operatorname{rank}\left(Z_{3}^{H} \mathcal{N}_{2}\left[\begin{array}{ccc}
I_{n} & 0 & 0
\end{array}\right]^{H}\right)=\operatorname{rank}\left(\left[\begin{array}{ccc}
0 & -1 & 0 \\
0 & 1 & 1
\end{array}\right]\right)=2=a_{\mu}, \\
& \operatorname{rank}\left(Z_{2}^{H} \mathcal{L}_{2}\left[\begin{array}{lll}
I_{n} & 0 & 0
\end{array}\right]^{H}\right)=\operatorname{rank}\left(\left[\begin{array}{ccc}
0 & 1 & 1
\end{array}\right]\right)=1=d_{\mu}^{(1)}+c_{0}+c_{1}, \\
& \operatorname{rank}\left(Z_{2}^{H} \mathcal{L}_{2}\left[\begin{array}{lll}
I_{n} & 0 & 0
\end{array}\right]^{H} T_{3}\right)=\operatorname{rank}\left(\left[\begin{array}{ll}
0 & ]
\end{array}\right)=0=d_{\mu}^{(1)},\right. \\
& \operatorname{rank}\left(M T_{3} T_{2}\right)=\operatorname{rank}\left(\left[\begin{array}{lll}
t & 0 & 0
\end{array}\right]^{H}\right)=1=d_{\mu}^{(2)}
\end{aligned}
$$

Finally, choosing $Z_{0}^{H}=\left[\begin{array}{lll}1 & 0 & 0\end{array}\right]$ we get a strangeness-free system of the form

$(3.8)\left[\begin{array}{lll}t & 0 & 0 \\ 0 & 0 & 0 \\ 0 & 0 & 0\end{array}\right] \ddot{x}+\left[\begin{array}{lll}1 & 0 & 0 \\ 0 & 0 & 0 \\ 0 & 0 & 0\end{array}\right] \dot{x}+\left[\begin{array}{ccc}1 & 0 & 0 \\ 0 & -1 & 0 \\ 0 & 1 & 1\end{array}\right] x=\left[\begin{array}{c}f_{1} \\ -f_{2}-2 \dot{f}_{2}-t \ddot{f}_{2}+\ddot{f}_{3} \\ -t f_{2}+f_{3}\end{array}\right]$,

with the same solution as the original system (1.3).

REMARK 3.9. The proof of Theorem 3.4 (and consequently, those of Corollary 3.5, Theorem 3.6 and Theorem 3.7) is given only for DAEs of strangeness index $\mu \leq 2$ for ease of presentation. However, the results are also valid for systems of arbitrary high index. To prove the results for linear second order systems (1.1) of arbitrary strangeness index $\mu>2$, the construction of a global canonical form analogous to the form given in [11, Theorem 3.21] is more convenient, but until now it is not clear how a suitable condensed form can be constructed.

REMARK 3.10. The derivative array approach presented in this section can also be extended to arbitrary linear high order differential-algebraic systems. The inflated system corresponding to (3.1) can be obtained in the same way by differentiating the original $k$-th order system and ordering the derivatives of the coefficient matrices in such a way that only the leading coefficient matrix has a lower triangular block structure and all other coefficient matrices of the inflated system have entries only in the first block columns. Then the results of Theorem 3.3 also hold for $k$-th order systems, and a hypothesis similar to Theorem 3.6 can be formulated so that it allows an index reduction for linear $k$-th order systems by choosing suitable projections in the same way as for linear second order systems. For the theoretical analysis of linear $k$-th order differential-algebraic systems, see also [12, 15]. Furthermore, a corresponding hypothesis for nonlinear second order differential-algebraic systems has been formulated in [18]. 
4. Order reduction for linear second order DAEs. The numerical solution of higher order differential-algebraic systems either requires the direct numerical solution of the higher order system by appropriate numerical methods (see, for example, $[14,17])$ or a suitable transformation into a first order system that does not increase the index. Since most of the numerical methods suited for the solution of DAEs are constructed for first order systems and these methods are well-studied-in general a transformation into a first order system is desired. Furthermore, for a robust solution, the numerical methods require differential-algebraic systems of low index such that besides the order reduction also an index reduction is required. In Example 1.1 we have seen that for higher order DAEs the classical order reduction by introducing new variables $v=\dot{x}$ for the derivatives can lead to an increase in the index of the DAE corresponding to higher smoothness requirements for the inhomogeneity $f(t)$ that even can cause the loss of solvability of the system; see also [12]. For $k$-th order linear DAEs it has been shown in [12] that if $\mu$ is the strangeness index of the triple of matrix-valued functions associated with the $k$-th order DAE system, then the maximal possible increase in the strangeness index $\tilde{\mu}$ of the first order system, obtained by the classical order reduction procedure, is $\tilde{\mu} \leq \mu+k-1$. In [12] it has been proposed to use the strangeness-free condensed form (2.4) given in Theorem 2.4 for the identification of those second order derivatives of variables that can be replaced to obtain a first order system that is strangeness-free without increasing the index. By introducing the new variable $\tilde{v}=\dot{\tilde{x}}_{1}$ for the strangeness-free system (2.4) we obtain a first order system in the variables $\left(\tilde{x}_{1}, \tilde{x}_{2}, \tilde{x}_{3}, \tilde{x}_{4}, \tilde{v}\right)$ that is also strangeness-free. The drawback of this approach is that there is no computationally feasible method to compute the condensed form (2.4), except if the structure can be used, since the derivatives of computed transformation matrices are used during the transformations. Further, the strangeness-free system (2.4) does not have the same solution $x$ as the original second order system (1.1), but a transformed solution $\tilde{x}=Q^{-1} x$. Nevertheless, the results suggest that applying index reduction first and then order reduction is a proper treatment of second order systems.

In the following, we will use the index reduction based on derivative arrays derived in Section 3 to obtain a strangeness-free second order system (3.7), which can then be used to construct a trimmed first order formulation in a numerical feasible way. We assume that we have locally computed a strangeness-free second order system (3.7) with matrix triple of the form

$$
(\hat{M}, \hat{C}, \hat{K})=\left(\left[\begin{array}{c}
\hat{M}_{1} \\
0 \\
0 \\
0
\end{array}\right],\left[\begin{array}{c}
\hat{C}_{1} \\
\hat{C}_{2} \\
0 \\
0
\end{array}\right],\left[\begin{array}{c}
\hat{K}_{1} \\
\hat{K}_{2} \\
\hat{K}_{3} \\
0
\end{array}\right]\right) .
$$

To find a suitable first order formulation, we first have to identify the second 
order differential variables. As the matrices $\hat{M}_{1}, \hat{C}_{2}$ and $\hat{K}_{3}$ have full row rank due to construction (see Theorem 3.6) there exists a pointwise unitary matrix-valued function $Q \in C\left(\mathbb{I}, \mathbb{C}^{n \times n}\right)$ that is sufficiently smooth such that

$$
\left[\begin{array}{c}
\hat{M}_{1} \\
\hat{C}_{2} \\
\hat{K}_{3}
\end{array}\right] Q=\left[\begin{array}{cccc}
M_{11} & 0 & 0 & 0 \\
C_{21} & C_{22} & 0 & 0 \\
K_{31} & K_{32} & K_{33} & 0
\end{array}\right],
$$

where the matrix-valued functions $M_{11}$ of size $d_{\mu}^{(2)} \times d_{\mu}^{(2)}, C_{22}$ of size $d_{\mu}^{(1)} \times d_{\mu}^{(1)}$ and $K_{33}$ of size $a_{\mu} \times a_{\mu}$ are pointwise nonsingular. With the corresponding basis transformation

$$
x=Q \hat{x}, \quad \dot{x}=Q \dot{\hat{x}}+\dot{Q} \hat{x}, \quad \ddot{x}=Q \ddot{\hat{x}}+2 \dot{Q} \dot{\hat{x}}+\ddot{Q} \hat{x},
$$

we get the equivalent system

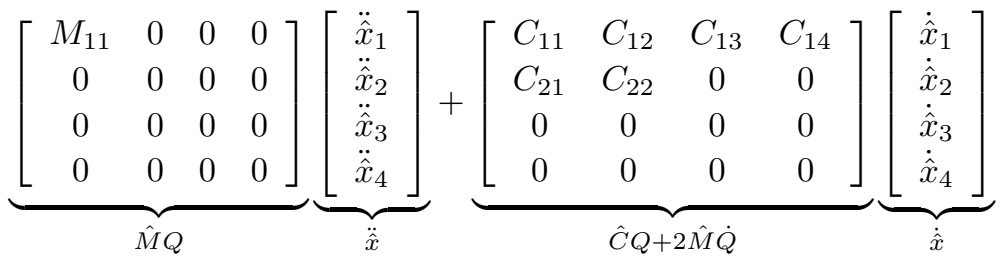

$$
\begin{aligned}
& +\underbrace{\left[\begin{array}{cccc}
K_{11} & K_{12} & K_{13} & K_{14} \\
K_{21} & K_{22} & K_{23} & K_{24} \\
K_{31} & K_{32} & K_{33} & 0 \\
0 & 0 & 0 & 0
\end{array}\right]}_{\hat{K} Q+\hat{C} \dot{Q}+\hat{M} \ddot{Q}} \underbrace{\left[\begin{array}{c}
\hat{x}_{1} \\
\hat{x}_{2} \\
\hat{x}_{3} \\
\hat{x}_{4}
\end{array}\right]}_{\hat{x}}=\underbrace{\left[\begin{array}{c}
\hat{f}_{1} \\
\hat{f}_{2} \\
\hat{f}_{3} \\
\hat{f}_{4}
\end{array}\right]}_{\hat{f}},
\end{aligned}
$$

where the second order differential variables $\hat{x}_{i}$ are explicitly specified. By introducing the new variable $\hat{v}=\dot{\hat{x}}_{1}$ we can transform the system (4.2) into first order form

$$
\begin{gathered}
{\left[\begin{array}{ccccc}
M_{11} & C_{11} & C_{12} & C_{13} & C_{14} \\
0 & C_{21} & C_{22} & 0 & 0 \\
0 & 0 & 0 & 0 & 0 \\
0 & 0 & 0 & 0 & 0 \\
0 & I & 0 & 0 & 0
\end{array}\right]\left[\begin{array}{c}
\dot{\hat{v}} \\
\dot{\hat{x}}_{1} \\
\dot{\hat{x}}_{2} \\
\dot{\hat{x}}_{3} \\
\dot{\hat{x}}_{4}
\end{array}\right]} \\
+\left[\begin{array}{ccccc}
0 & K_{11} & K_{12} & K_{13} & K_{14} \\
0 & K_{21} & K_{22} & K_{23} & K_{24} \\
0 & K_{31} & K_{32} & K_{33} & 0 \\
0 & 0 & 0 & 0 & 0 \\
-I & 0 & 0 & 0 & 0
\end{array}\right]\left[\begin{array}{c}
\hat{v} \\
\hat{x}_{1} \\
\hat{x}_{2} \\
\hat{x}_{3} \\
\hat{x}_{4}
\end{array}\right]=\left[\begin{array}{c}
\hat{f}_{1} \\
\hat{f}_{2} \\
\hat{f}_{3} \\
\hat{f}_{4} \\
0
\end{array}\right]
\end{gathered}
$$


Here, we have

$\hat{v}=\left[\begin{array}{llll}I & 0 & 0 & 0\end{array}\right] \dot{\hat{x}}=\left[\begin{array}{llll}I & 0 & 0 & 0\end{array}\right]\left(\dot{Q}^{H} x+Q^{H} \dot{x}\right)=\dot{Q}_{1}^{H} x+Q_{1}^{H} \dot{x}=\frac{d}{d t}\left(Q_{1}^{H} x\right)$,

$\dot{\hat{v}}=\frac{d^{2}}{d t^{2}}\left(Q_{1}^{H} x\right)$,

with $Q_{1}=Q\left[\begin{array}{llll}I & 0 & 0 & 0\end{array}\right]^{H}$. Therefore, system (4.3) is equivalent to

$$
\begin{aligned}
& {\left[\begin{array}{cc}
\hat{M} Q_{1} & \hat{C} Q+2 \hat{M} \dot{Q} \\
0 & J
\end{array}\right]\left[\begin{array}{c}
\frac{d^{2}}{d t^{2}}\left(Q_{1}^{H} x\right) \\
\frac{d}{d t}\left(Q^{H} x\right)
\end{array}\right]} \\
& \quad+\left[\begin{array}{cc}
0 & \hat{K} Q+\hat{C} \dot{Q}+\hat{M} \ddot{Q} \\
-I & 0
\end{array}\right]\left[\begin{array}{c}
\frac{d}{d t}\left(Q_{1}^{H} x\right) \\
Q^{H} x
\end{array}\right]=\left[\begin{array}{c}
\hat{f} \\
0
\end{array}\right],
\end{aligned}
$$

with $J=\left[\begin{array}{llll}I & 0 & 0 & 0\end{array}\right]$. Now, by introducing another variable

$$
v=Q_{1}^{H} \dot{x}=\frac{d}{d t}\left(Q_{1}^{H} x\right)-\dot{Q}_{1}^{H} x,
$$

the first equation of (4.4) becomes

$$
\hat{M} Q_{1} \dot{v}+\left(\hat{C}+\hat{M}\left(Q_{1} \dot{Q}_{1}^{H}+2 \dot{Q} Q^{H}\right)\right) \dot{x}+\left(\hat{K}+\hat{M}\left(Q_{1} \ddot{Q}_{1}^{H}+2 \dot{Q} \dot{Q}^{H}+\ddot{Q} Q^{H}\right)\right) x=\hat{f},
$$

where we have used that $\dot{Q} Q^{H}+Q \dot{Q}^{H}=0$, as $Q$ is unitary. Thus, we get a first order system in the original variable $x$ and in $v$ of the form

$$
\left[\begin{array}{cc}
\hat{M} Q_{1} & \tilde{C} \\
0 & Q_{1}^{H}
\end{array}\right]\left[\begin{array}{c}
\dot{v} \\
\dot{x}
\end{array}\right]+\left[\begin{array}{cc}
0 & \tilde{K} \\
-I & 0
\end{array}\right]\left[\begin{array}{l}
v \\
x
\end{array}\right]=\left[\begin{array}{l}
\hat{f} \\
0
\end{array}\right]
$$

with

$$
\begin{aligned}
& \tilde{C}=\hat{C}+\hat{M}\left(Q_{1} \dot{Q}_{1}^{H}+2 \dot{Q} Q^{H}\right)=\hat{C}+\hat{M}\left(Q_{1} \dot{Q}_{1}^{H}-2 Q \dot{Q}^{H}\right), \\
& \tilde{K}=\hat{K}+\hat{M}\left(Q_{1} \ddot{Q}_{1}^{H}+2 \dot{Q} \dot{Q}^{H}+\ddot{Q} Q^{H}\right)=\hat{K}+\hat{M}\left(Q_{1} \ddot{Q}_{1}^{H}-Q \ddot{Q}^{H}\right),
\end{aligned}
$$

using that $\ddot{Q} Q^{H}+2 \dot{Q} \dot{Q}^{H}+Q \ddot{Q}^{H}=0$. In addition, it holds that

$$
\begin{gathered}
Q_{1} \dot{Q}_{1}^{H}-2 Q \dot{Q}^{H}=Q J^{H} J \dot{Q}^{H}-2 Q \dot{Q}^{H}=Q\left[\begin{array}{cccc}
-I & 0 & 0 & 0 \\
0 & -2 I & 0 & 0 \\
0 & 0 & -2 I & 0 \\
0 & 0 & 0 & -2 I
\end{array}\right] \dot{Q}^{H}, \\
Q_{1} \ddot{Q}_{1}^{H}-Q \ddot{Q}^{H}=Q J^{H} J \ddot{Q}^{H}-Q \ddot{Q}^{H}=Q\left[\begin{array}{cccc}
0 & 0 & 0 & 0 \\
0 & -I & 0 & 0 \\
0 & 0 & -I & 0 \\
0 & 0 & 0 & -I
\end{array}\right] \ddot{Q}^{H},
\end{gathered}
$$


and

$$
\begin{aligned}
& \tilde{C}=\hat{C}-\hat{M} Q\left[\begin{array}{cccc}
I & 0 & 0 & 0 \\
0 & 2 I & 0 & 0 \\
0 & 0 & 2 I & 0 \\
0 & 0 & 0 & 2 I
\end{array}\right] \dot{Q}^{H}=\hat{C}-\left[\begin{array}{cccc}
M_{11} & 0 & 0 & 0 \\
0 & 0 & 0 & 0 \\
0 & 0 & 0 & 0 \\
0 & 0 & 0 & 0
\end{array}\right] \dot{Q}^{H}=\hat{C}-\hat{M} Q \dot{Q}^{H}, \\
& \tilde{K}=\hat{K}-\hat{M} Q\left[\begin{array}{llll}
0 & 0 & 0 & 0 \\
0 & I & 0 & 0 \\
0 & 0 & I & 0 \\
0 & 0 & 0 & I
\end{array}\right] \ddot{Q}^{H}=\hat{K} .
\end{aligned}
$$

Altogether, we have constructed a first order formulation in the original variable $x$ and $v$, only using the coefficient matrices of the strangeness-free formulation (3.7) and the unitary transformation matrix $Q$. Due to construction this system is strangenessfree.

THEOREM 4.1. Consider a strangeness-free linear second order differentialalgebraic system (3.7) with matrix-valued functions $\hat{M}, \hat{C}, \hat{K} \in C\left(\mathbb{I}, \mathbb{C}^{m, n}\right)$ and righthand side $\hat{f} \in C\left(\mathbb{I}, \mathbb{C}^{m}\right)$. Further, let $Q \in C^{1}\left(\mathbb{I}, \mathbb{C}^{n, n}\right)$ be a unitary matrix-valued function that decomposes $\hat{M}, \hat{C}, \hat{K}$ as in (4.1). Then the trimmed first order formulation

$$
\left[\begin{array}{cc}
\hat{M} Q_{1} & \hat{C}+\hat{M} \dot{Q} Q^{H} \\
0 & Q_{1}^{H}
\end{array}\right]\left[\begin{array}{l}
\dot{v} \\
\dot{x}
\end{array}\right]+\left[\begin{array}{cc}
0 & \hat{K} \\
-I & 0
\end{array}\right]\left[\begin{array}{l}
v \\
x
\end{array}\right]=\left[\begin{array}{l}
\hat{f} \\
0
\end{array}\right]
$$

is also strangeness-free, with $Q_{1}=Q\left[\begin{array}{llll}I & 0 & 0 & 0\end{array}\right]^{H}$, and the characteristic values are given by $d_{\mu}=2 d_{\mu}^{(2)}+d_{\mu}^{(1)}, a_{\mu}, v_{\mu}$ and $u_{\mu}$.

Proof. The proof follows directly from the construction of the trimmed first order formulation (4.5). Setting $\hat{x}=Q^{H} x$ and $\hat{v}=\frac{d}{d t}\left(Q_{1}^{H} x\right)=v+\dot{Q}_{1}^{H} x$, we obtain

$$
\begin{aligned}
& {\left[\begin{array}{ccccc}
M_{11} & C_{11} & C_{12} & C_{13} & C_{14} \\
0 & C_{21} & C_{22} & 0 & 0 \\
0 & 0 & 0 & 0 & 0 \\
0 & 0 & 0 & 0 & 0 \\
0 & I_{d_{\mu}^{(2)}} & 0 & 0 & 0
\end{array}\right]\left[\begin{array}{c}
\dot{\hat{v}} \\
\hat{\hat{x}}_{1} \\
\dot{\hat{x}}_{2} \\
\dot{\hat{x}}_{3} \\
\dot{\hat{x}}_{4}
\end{array}\right]} \\
& +\left[\begin{array}{ccccc}
0 & K_{11} & K_{12} & K_{13} & K_{14} \\
0 & K_{21} & K_{22} & K_{23} & K_{24} \\
0 & K_{31} & K_{32} & K_{33} & 0 \\
0 & 0 & 0 & 0 & 0 \\
-I_{d_{\mu}^{(2)}} & 0 & 0 & 0 & 0
\end{array}\right]\left[\begin{array}{c}
\hat{v} \\
\hat{x}_{1} \\
\hat{x}_{2} \\
\hat{x}_{3} \\
\hat{x}_{4}
\end{array}\right]=\left[\begin{array}{c}
\hat{f}_{1} \\
\hat{f}_{2} \\
\hat{f}_{3} \\
\hat{f}_{4} \\
0
\end{array}\right],
\end{aligned}
$$

which is clearly strangeness-free, since $M_{11}, C_{22}$ and $K_{33}$ are nonsingular.

Theorem 4.1 allows the construction of a first order formulation directly from the coefficients of the strangeness-free second order system (3.7). The trimmed first order 
formulation (4.5) is of minimal possible size and has the same solution component $x$ as the original system (1.1), and no further smoothness requirements for the inhomogeneity are required. Since the first order formulation (4.5) is strangeness-free, numerical methods suited for differential-algebraic systems can be directly applied to the first order system (4.5).

EXAMPLE 4.2. For the second order system (1.3) given in Example 1.1 we have computed an equivalent strangeness-free formulation (3.8) using the derivative array approach. Following Theorem 4.1 a trimmed first order formulation (with $Q=I$ ) for system (1.3) is given by

$$
\left[\begin{array}{c|ccc}
t & 1 & 0 & 0 \\
0 & 0 & 0 & 0 \\
0 & 0 & 0 & 0 \\
\hline 0 & 1 & 0 & 0
\end{array}\right]\left[\begin{array}{c}
\dot{v} \\
\dot{x}_{1} \\
\dot{x}_{2} \\
\dot{x}_{3}
\end{array}\right]+\left[\begin{array}{c|ccc}
0 & 1 & 0 & 0 \\
0 & 0 & -1 & 0 \\
0 & 0 & 1 & 1 \\
\hline-1 & 0 & 0 & 0
\end{array}\right]\left[\begin{array}{c}
v \\
x_{1} \\
x_{2} \\
x_{3}
\end{array}\right]=\left[\begin{array}{c}
f_{1} \\
-f_{2}-2 \dot{f}_{2}-t \ddot{f}_{2}+\ddot{f}_{3} \\
-t f_{2}+f_{3} \\
0
\end{array}\right],
$$

which has the same solution components $x_{1}, x_{2}$, and $x_{3}$ as the original system (1.3).

REMARK 4.3. For strangeness-free linear $k$-th order system, the trimmed order reduction formalism can also be applied successively to the $k$-th order system to reduce the order by one in each reduction step. In this process the derivative of order $(k-1)$ of the transformation matrix $Q$, chosen similar as in (4.1), will occur. In the constant coefficient case structure preserving staircase forms for matrix triples are given in [3], that allow trimmed linearizations for arbitrary high order systems in the context of matrix polynomials. For the variable coefficient case, however, it is not clear if such structure preserving staircase forms exist and how trimmed first order formulations can be derived in this case.

5. Conclusions. In this paper, we have discussed the solution of linear second order differential-algebraic equations with variable coefficients. Since index reduction and order reduction for higher order higher index DAEs do not commute, appropriate index reduction methods for higher order DAEs are required. We have presented a numerically computable way to determine a strangeness-free normal form using the derivative array approach for linear second order DAEs. For differentialalgebraic systems with well-defined strangeness index $\mu$ the complete structural information on the global characteristic values of the triple $(M(t), C(t), K(t))$ can be obtained from the local information of an inflated triples and it is possible to derive a strangeness-free differential-algebraic system using only local information. For this strangeness-free second order system, a trimmed first order formulation is derived, which is strangeness-free and has the same solution components as the original second order system. In conclusion, for an appropriate treatment of higher order differential-algebraic system, the index reduction should be carried out at first either by transforming into the condensed form (2.4) or by using the derivative arrays (3.1), 
followed possibly by a suitable order reduction to obtain a strangeness-free first order system.

\section{REFERENCES}

[1] C. Arévalo and P. Lötstedt. Improving the accuracy of BDF methods for index 3 differentialalgebraic equations. BIT Numerical Mathematics, 35:297-308, 1994.

[2] K.E. Brenan, S.L. Campbell, and L.R. Petzold. Numerical Solution of Initial-Value Problems in Differential Algebraic Equations. SIAM, Classics in Applied Mathematics 14, Philadelphia, PA, 1996.

[3] R. Byers, V. Mehrmann, and H. Xu. A structured staircase algorithm for skewsymmetric/symmetric pencils. Electronic Transactions on Numerical Analysis, 26:1-33, 2007.

[4] S.L. Campbell. Comment on controlling generalized state-space (descriptor) systems. International Journal on Control, 46:2229-2230, 1987.

[5] C. De Boor and H.-O. Kreiss. On the condition of the linear systems associated with discretized BVPs of ODEs. SIAM Journal on Numerical Analysis, 23(5):936-939, 1986.

[6] E. Eich-Soellner and C. Führer. Numerical Methods in Multibody Dynamics. B.G. Teubner, Stuttgart, 1998.

[7] G.H. Golub and C.F. van Loan. Matrix Computations, third edition. The Johns Hopkins University Press, Baltimore and London, 1996.

[8] M. Günther and U. Feldmann. CAD-based electric-circuit modeling in industry, I. Mathematical structure and index of network equations. Surveys on Mathematics for Industry, 8:97-129, 1999.

[9] M. Günther and U. Feldmann. CAD-based electric-circuit modeling in industry, II. Impact of circuit configuration and parameters. Surveys on Mathematics for Industry, 8:131-157, 1999.

[10] E. Hairer and G. Wanner. Solving Ordinary Differential Equations II: Stiff and DifferentialAlgebraic Problems, second edition. Springer-Verlag, Berlin, 1996.

[11] P. Kunkel and V. Mehrmann. Differential-Algebraic Equations - Analysis and Numerical Solution. EMS Publishing House, Zürich, 2006.

[12] V. Mehrmann and C. Shi. Transformation of high order linear differential-algebraic systems to first order. Numerical Algorithms, 42(3-4):281-307, 2006.

[13] P.J. Rabier and W.C. Rheinboldt. Nonholonomic Motion of Rigid Mechanical Systems from a DAE Viewpoint. SIAM, Philadelphia, PA 19104-2688, 2000.

[14] J. Sand. On implicit Euler and related methods for high-order high-index DAEs. Applied Numerical Mathematics, 42:411-424, 2002.

[15] C. Shi. Linear Differential-Algebraic Equations of Higher-Order and the Regularity or Singularity of Matrix Polynomials. PhD Thesis, Institut für Mathematik, Technische Universität Berlin, 2004.

[16] L. Wunderlich. Numerical Solution of Second Order Differential-Algebraic Equations. Diploma Thesis, Institut für Mathematik, Technische Universität Berlin, 2004.

[17] L. Wunderlich. Numerical Solution of Semi-Explicit Systems of Second Order DifferentialAlgebraic Equations. Preprint 24, Institut für Mathematik, Technische Universität Berlin, 2005.

[18] L. Wunderlich. Analysis and Numerical Solution of Structured and Switched DifferentialAlgebraic Systems. PhD Thesis, Institut für Mathematik, Technische Universität Berlin, 2008.

[19] L. Wunderlich. A derivative array approach for linear second order differential-algebraic systems. DFG Research Center Matheon, Mathematics for key technologies in Berlin, Technical Report 529, Technische Universität Berlin, Germany, 2008. 


\section{Appendix A.}

For triples $(M, C, K)$ of matrix-valued functions $M, C, K \in C\left(\mathbb{I}, \mathbb{C}^{m \times n}\right)$, a global condensed form under global equivalence transformations (2.1) has been derived in $[12,15]$.

Lemma A.1. $[12,15]$ Let the matrix-valued functions $M, C, K \in C\left(\mathbb{I}, \mathbb{C}^{m \times n}\right)$ be sufficiently smooth, and suppose that the regularity conditions (2.3) hold for the local characteristic values of $(M, C, K)$. Then $(M, C, K)$ is globally equivalent to a triple of matrix-valued functions $(\tilde{M}, \tilde{C}, \tilde{K})$ of the condensed form

$$
\left(\left[\begin{array}{cccccccc}
I_{s}(M C K) & 0 & 0 & 0 & 0 & 0 & 0 & 0 \\
0 & I_{s}(M C) & 0 & 0 & 0 & 0 & 0 & 0 \\
0 & 0 & I_{s}(M K) & 0 & 0 & 0 & 0 & 0 \\
0 & 0 & 0 & I_{d^{(2)}} & 0 & 0 & 0 & 0 \\
0 & 0 & 0 & 0 & 0 & 0 & 0 & 0 \\
0 & 0 & 0 & 0 & 0 & 0 & 0 & 0 \\
0 & 0 & 0 & 0 & 0 & 0 & 0 & 0 \\
0 & 0 & 0 & 0 & 0 & 0 & 0 & 0 \\
0 & 0 & 0 & 0 & 0 & 0 & 0 & 0 \\
0 & 0 & 0 & 0 & 0 & 0 & 0 & 0 \\
0 & 0 & 0 & 0 & 0 & 0 & 0 & 0 \\
0 & 0 & 0 & 0 & 0 & 0 & 0 & 0 \\
0 & 0 & 0 & 0 & 0 & 0 & 0 & 0
\end{array}\right],\right.
$$

$$
\left[\begin{array}{cccccccc}
0 & 0 & C & C & 0 & 0 & C & C \\
0 & 0 & C & C & 0 & 0 & C & C \\
0 & 0 & C & C & 0 & 0 & C & C \\
0 & 0 & C & C & 0 & 0 & C & C \\
0 & 0 & 0 & 0 & I_{s}(C K) & 0 & 0 & 0 \\
0 & 0 & 0 & 0 & 0 & I_{d^{(1)}} & 0 & 0 \\
I_{s(M C K)} & 0 & 0 & 0 & 0 & 0 & 0 & 0 \\
0 & I_{s}(M C) & 0 & 0 & 0 & 0 & 0 & 0 \\
0 & 0 & 0 & 0 & 0 & 0 & 0 & 0 \\
0 & 0 & 0 & 0 & 0 & 0 & 0 & 0 \\
0 & 0 & 0 & 0 & 0 & 0 & 0 & 0 \\
0 & 0 & 0 & 0 & 0 & 0 & 0 & 0 \\
0 & 0 & 0 & 0 & 0 & 0 & 0 & 0
\end{array}\right],
$$




$\left.\left[\begin{array}{cccccccc}0 & K & 0 & K & 0 & K & 0 & K \\ 0 & K & 0 & K & 0 & K & 0 & K \\ 0 & K & 0 & K & 0 & K & 0 & K \\ 0 & K & 0 & K & 0 & K & 0 & K \\ 0 & K & 0 & K & 0 & K & 0 & K \\ 0 & K & 0 & K & 0 & K & 0 & K \\ 0 & K & 0 & K & 0 & K & 0 & K \\ 0 & K & 0 & K & 0 & K & 0 & K \\ 0 & 0 & 0 & 0 & 0 & 0 & I_{a} & 0 \\ 0 & 0 & 0 & 0 & I_{s}(C K) & 0 & 0 & 0 \\ 0 & 0 & I_{s}(M K) & 0 & 0 & 0 & 0 & 0 \\ I_{s(M C K)} & 0 & 0 & 0 & 0 & 0 & 0 & 0 \\ 0 & 0 & 0 & 0 & 0 & 0 & 0 & 0\end{array}\right]\right) \begin{aligned} & s^{(M C K)} \\ & s^{(M C)} \\ & s^{(M K)} \\ & d^{(2)} \\ & s^{(C K)} \\ & d^{(1)} \\ & s^{(M C K)} \\ & s^{(M C)} \\ & a \\ & s^{(C K)} \\ & s^{(M K)} \\ & s^{(M C K)} \\ & v\end{aligned}$

Here, all blocks are again functions on $\mathbb{I}$ and the last block columns have size $u$. 


\section{Appendix B.}

Proof of Theorem 3.6. By assumption, the strangeness index is well-defined and the ranks of $\mathcal{M}_{\mu}, \mathcal{L}_{\mu}$ and $\mathcal{N}_{\mu}$ are constant on $\mathbb{I}$ with

$$
\begin{aligned}
& \operatorname{rank}\left[\mathcal{M}_{\mu}, \mathcal{L}_{\mu}, \mathcal{N}_{\mu}\right]=(\mu+1) m-\tilde{v}_{\mu}, \\
& \operatorname{rank}\left[\mathcal{M}_{\mu}, \mathcal{L}_{\mu}\right]=(\mu+1) m-a_{\mu}-\tilde{v}_{\mu}, \\
& \operatorname{rank} \mathcal{M}_{\mu}=(\mu+1) m-a_{\mu}-\tilde{v}_{\mu}-d_{\mu}^{(1)}-\sum_{i=0}^{\mu-1} c_{i},
\end{aligned}
$$

due to Theorem 3.4 and Corollary 3.5 (see also the relations (3.5)). Thus, there exists a continuous matrix-valued function $Z$ of size $\left((\mu+1) m, a_{\mu}+\tilde{v}_{\mu}+d_{\mu}^{(1)}+\sum_{i=0}^{\mu-1} c_{i}\right)$ whose columns form a basis of corange $\mathcal{M}_{\mu}$, i.e., $Z^{H} \mathcal{M}_{\mu}=0$. Without loss of generality the matrix $Z$ can be partitioned into $Z=\left[Z_{2}, Z_{3}, Z_{4}\right]$, with $Z_{2}$ of size $\left((\mu+1) m, d_{\mu}^{(1)}+\right.$ $\left.\sum_{i=0}^{\mu-1} c_{i}\right), Z_{3}$ of size $\left((\mu+1) m, a_{\mu}\right)$ and $Z_{4}$ of size $\left((\mu+1) m, \tilde{v}_{\mu}\right)$ such that

$$
Z_{3}^{H} \mathcal{L}_{\mu}=0, \quad Z_{4}^{H} \mathcal{L}_{\mu}=0, \quad Z_{4}^{H} \mathcal{N}_{\mu}=0,
$$

i.e., the columns of the matrices $Z_{4}$ and $Z_{3}$ form bases of corange $\left(\left[\mathcal{M}_{\mu}, \mathcal{L}_{\mu}, \mathcal{N}_{\mu}\right]\right)$, and of corange $\left(\left[\mathcal{M}_{\mu}, \mathcal{L}_{\mu}\right]\right)$, respectively. First, we note that multiplication of (3.1) for $l=\mu$ by $Z_{3}^{H}$ gives

$$
Z_{3}^{H} \mathcal{N}_{\mu} z_{\mu}=Z_{3}^{H} g_{\mu}
$$

The only nontrivial entries in $\mathcal{N}_{\mu}$ are in the first block column belonging to the original unknown $x$. Hence, we get purely algebraic equations for $x$. Lemma 2.3 and (3.4) give

$$
\operatorname{rank}\left(Z_{3}^{H} \mathcal{N}_{\mu}\left[\begin{array}{cccc}
I_{n} & 0 & \ldots & 0
\end{array}\right]^{H}\right)=\tilde{a}_{\mu}+\tilde{s}_{\mu}^{(M C K)}+\tilde{s}_{\mu}^{(C K)}+\tilde{s}_{\mu}^{(M K)}=a_{\mu},
$$

thus, with $Z_{3}$ we obtain the complete set of algebraic equations. Next, we must get $d_{\mu}^{(1)}$ first order differential equations and $d_{\mu}^{(2)}$ second order differential equations to complete these algebraic equations to a strangeness-free differential-algebraic system. In a similar way, multiplication of (3.1) with the matrix $Z_{2}^{H}$ yields

$$
Z_{2}^{H} \mathcal{L}_{\mu} \dot{z}_{\mu}+Z_{2}^{H} \mathcal{N}_{\mu} z_{\mu}=Z_{2}^{H} g_{\mu} .
$$

Again, the only non-zero entries of $\mathcal{L}_{\mu}$ are in the first block column belonging to the first order derivative $\dot{x}$. Lemma 2.3 and (3.4) give

$$
\operatorname{rank}\left(Z_{2}^{H} \mathcal{L}_{\mu}\right)=\tilde{d}_{\mu}^{(1)}+\tilde{s}_{\mu}^{(C K)}+\tilde{s}_{\mu}^{(M C)}+\tilde{s}_{\mu}^{(M C K)}=\sum_{i=0}^{\mu} q_{i}=d_{\mu}^{(1)}+\sum_{i=0}^{\mu-1} c_{i} .
$$

So far we have shown the first three parts of Theorem 3.6. To show part 4 and 5 of the Theorem we treat systems of strangeness index $\mu=0,1$ and 2 separately. Let 
$(\tilde{M}, \tilde{C}, \tilde{K})$ be a normal form of the triple $(M, C, K)$ according to the global condensed form (A.1) with corresponding inflated triples $\left(\tilde{\mathcal{M}}_{\mu}, \tilde{\mathcal{L}}_{\mu}, \tilde{\mathcal{N}}_{\mu}\right)$. Due to Theorem 3.3, there exist matrices $\Pi, \Theta, \Psi$, and $\Sigma$ such that

$$
\tilde{\mathcal{M}}_{\mu}=\Pi \mathcal{M}_{\mu} \Theta, \quad \tilde{\mathcal{L}}_{\mu}=\Pi \mathcal{L}_{\mu} \Theta+2 \Pi \mathcal{M}_{\mu} \Psi, \quad \tilde{\mathcal{N}}_{\mu}=\Pi \mathcal{N}_{\mu} \Theta+\Pi \mathcal{L}_{\mu} \Psi+\Pi \mathcal{M}_{\mu} \Sigma
$$

according to (3.3). For $\mu=0$ the triple $\left(\tilde{\mathcal{M}}_{0}, \tilde{\mathcal{L}}_{0}, \tilde{\mathcal{N}}_{0}\right)$ is of the form

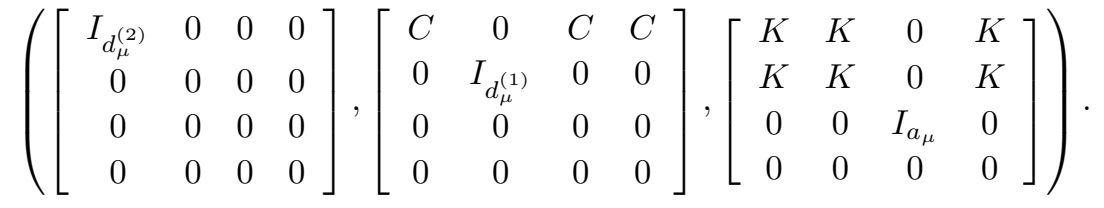

Let $\Pi$ and $\Theta$ be partitioned as $\Pi:=\left[\Pi_{1}^{H}, \Pi_{2}^{H}, \Pi_{3}^{H}, \Pi_{4}^{H}\right]^{H}$ and $\Theta:=\left[\Theta_{1}, \Theta_{2}, \Theta_{3}, \Theta_{4}\right]$ according to (B.1). Then setting

$$
Z_{2}=\Pi_{2}^{H}, \quad Z_{3}=\Pi_{3}^{H}, \quad Z_{4}=\Pi_{4}^{H}
$$

yields

$$
\begin{aligned}
& Z_{4}^{H} \mathcal{M}_{0}=0, \quad Z_{4}^{H} \mathcal{L}_{0}=0, \quad Z_{4}^{H} \mathcal{N}_{0}=0, \\
& Z_{3}^{H} \mathcal{M}_{0}=0, \quad Z_{3}^{H} \mathcal{L}_{0}=0, \quad Z_{2}^{H} \mathcal{M}_{0}=0,
\end{aligned}
$$

as well as

$$
\begin{aligned}
\operatorname{rank}\left(Z_{3}^{H} \mathcal{N}_{0}\right) & =\operatorname{rank}\left[\begin{array}{cccc}
0 & 0 & I_{a_{\mu}} & 0
\end{array}\right]=a_{\mu}, \\
\operatorname{rank}\left(Z_{2}^{H} \mathcal{L}_{0}\right) & =\operatorname{rank}\left[\begin{array}{llll}
0 & I_{d_{\mu}^{(1)}} & 0 & 0
\end{array}\right]=d_{\mu}^{(1)} .
\end{aligned}
$$

By setting $T_{3}=\left[\Theta_{1}, \Theta_{2}, \Theta_{4}\right]$, we get

$$
\operatorname{rank}\left(Z_{2}^{H} \mathcal{L}_{0} T_{3}\right)=\operatorname{rank}\left[\begin{array}{ccc}
0 & I_{d_{\mu}^{(1)}} & 0
\end{array}\right]=d_{\mu}^{(1)},
$$

and, with $Z_{1}=I_{d_{\mu}^{(1)}}$ and $T_{2}=\left[\begin{array}{cc}I_{d_{\mu}^{(2)}} & 0 \\ 0 & 0 \\ 0 & I_{u_{\mu}}\end{array}\right]$, we have $Z_{1}^{H} Z_{2}^{H} \mathcal{L}_{0} T_{3} T_{2}=0$, and

$$
\operatorname{rank}\left(M T_{3} T_{2}\right)=\operatorname{rank}\left[\begin{array}{cc}
I_{d_{\mu}^{(2)}} & 0 \\
0 & 0 \\
0 & 0 \\
0 & 0
\end{array}\right]=d_{\mu}^{(2)}
$$

Finally, setting $Z_{0}^{H}=\left[\begin{array}{llll}I_{d_{\mu}^{(2)}} & 0 & 0 & 0\end{array}\right]$ yields

$$
\operatorname{rank}\left(Z_{0}^{H} M\right)=\operatorname{rank}\left[\begin{array}{cccc}
I_{d_{\mu}^{(2)}} & 0 & 0 & 0
\end{array}\right]=d_{\mu}^{(2)} .
$$


In the case $\mu=1$ we have to consider the inflated triple

$$
\left(\tilde{\mathcal{M}}_{1}, \tilde{\mathcal{L}}_{1}, \tilde{\mathcal{N}}_{1}\right)=\left(\left[\begin{array}{cc}
\tilde{M} & 0 \\
\dot{\tilde{M}}+\tilde{C} & \tilde{M}
\end{array}\right],\left[\begin{array}{cc}
\tilde{C} & 0 \\
\dot{\tilde{C}}+\tilde{K} & 0
\end{array}\right],\left[\begin{array}{cc}
\tilde{K} & 0 \\
\dot{\tilde{K}} & 0
\end{array}\right]\right)
$$

The identity blocks in the matrix $\tilde{M}$ allow to eliminate all other entries in the corresponding block rows of $\tilde{\mathcal{M}}_{1}$ by local equivalence transformations. Further eliminations using the identity blocks of the global condensed form and block decompositions yield the following matrix triple, where we only state the first block columns of $\tilde{\mathcal{L}}_{1}$ and $\tilde{\mathcal{N}}_{1}$, since all other entries are zero:

(B.2)

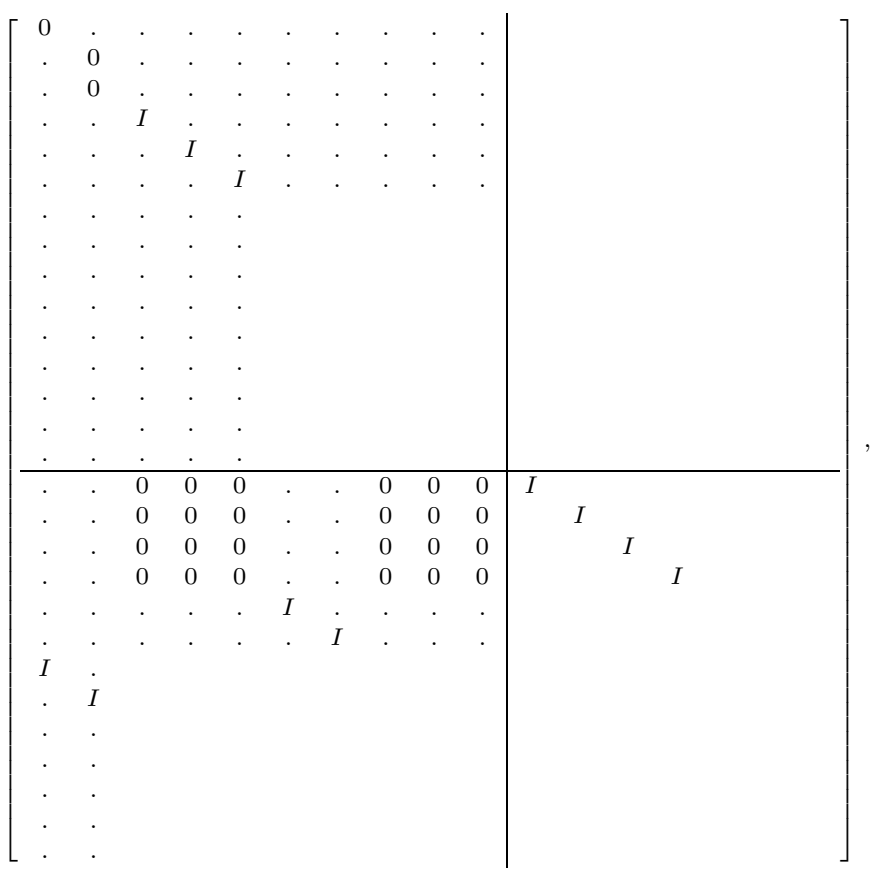


with dimensions $\tilde{s}_{1}=s_{0}^{(M C K)}, \tilde{s}_{2}=s_{0}^{(M C)}, \tilde{s}_{3}=s_{0}^{(M K)}, \tilde{s}_{4}=s_{0}^{(C K)}, \tilde{s}_{5}=s_{0}^{(M C K)}+$ $s_{0}^{(M C)}-e_{1}, \tilde{s}_{6}=s_{0}^{(M K)}-e_{1}+k_{1}, \tilde{e}_{1}=e_{1}-k_{1}, \tilde{d}_{1}=d_{0}^{(1)}$, and $\tilde{d}_{2}=d_{0}^{(2)}$. Thus, the triple $\left(\tilde{\mathcal{M}}_{1}, \tilde{\mathcal{L}}_{1}, \tilde{\mathcal{N}}_{1}\right)$ can be assumed to be in the form (B.2) and $\Pi$ and $\Theta$ can be partitioned as

$$
\Pi:=\left[\Pi_{1}^{H}, \ldots, \Pi_{28}^{H}\right]^{H}, \quad \Theta:=\left[\Theta_{1}, \ldots, \Theta_{18}\right]
$$

corresponding to the row and column structure of the block matrices in (B.2). Then we have

$$
\begin{aligned}
& {\left[\Pi_{1}^{H}, \Pi_{2}^{H}, \Pi_{3}^{H}, \Pi_{7}^{H}, \ldots, \Pi_{15}^{H}, \Pi_{24}^{H}, \ldots, \Pi_{28}^{H}\right]^{H} \mathcal{M}_{1}=0} \\
& \mathcal{M}_{1}\left[\Theta_{8}, \Theta_{9}, \Theta_{10}, \Theta_{15}, \ldots, \Theta_{18}\right]=0 \\
& {\left[\Pi_{3}^{H}, \Pi_{7}^{H}, \Pi_{9}^{H}, \Pi_{11}^{H}, \ldots, \Pi_{15}^{H}, \Pi_{28}^{H}\right]^{H} \mathcal{L}_{1}=0} \\
& {\left[\Pi_{1}^{H}, \Pi_{2}^{H}, \Pi_{3}^{H}, \Pi_{7}^{H}, \ldots, \Pi_{15}^{H}, \Pi_{24}^{H}, \ldots, \Pi_{28}^{H}\right]^{H} \mathcal{L}_{1}\left[\Theta_{10}, \Theta_{15}, \ldots, \Theta_{18}\right]=0}
\end{aligned}
$$

and set

$$
\begin{aligned}
Z & =\left[\Pi_{1}^{H}, \Pi_{2}^{H}, \Pi_{3}^{H}, \Pi_{7}^{H}, \ldots, \Pi_{15}^{H}, \Pi_{24}^{H}, \ldots, \Pi_{28}^{H}\right], \\
Z_{4} & =\left[\Pi_{3,2}^{H}, \Pi_{7,2}^{H}, \Pi_{9,2}^{H}, \Pi_{15}^{H}, \Pi_{28}^{H}\right], \\
Z_{3} & =\left[\Pi_{3,1}^{H}, \Pi_{7,1}^{H}, \Pi_{9,1}^{H}, \Pi_{11}^{H}, \ldots, \Pi_{14}^{H}\right], \\
Z_{2} & =\left[\Pi_{1}^{H}, \Pi_{2}^{H}, \Pi_{8}^{H}, \Pi_{10}^{H}, \Pi_{24}^{H}, \ldots, \Pi_{27}^{H}\right],
\end{aligned}
$$


where $\Pi_{., 1}$ and $\Pi_{., 2}$ and $\Theta_{., 1}$ and $\Theta_{., 2}$ denote the parts of the rows of $\Pi$. and columns of $\Theta$. that corresponds to the range and nullspace of block rows and block columns, respectively, after one more block decomposition of the matrices in (B.2). We have

$$
\begin{aligned}
& \operatorname{rank}\left(Z_{3}^{H} \mathcal{N}_{1}\right)=c_{0}+c_{1}=a_{\mu}, \\
& \operatorname{rank}\left(Z_{2}^{H} \mathcal{L}_{1}\right)=e_{1}+d_{0}^{(1)}+s_{0}^{(M C)}+c_{0}=d_{\mu}^{(1)}+c_{0} .
\end{aligned}
$$

Setting $T_{3}=\left[\Theta_{2,2}, \Theta_{4,2}, \Theta_{5,2}, \Theta_{7,2}, \Theta_{9,2}, \Theta_{10,2}\right]$, we get

$$
\operatorname{rank}\left(Z_{2}^{H} \mathcal{L}_{1}\left[\begin{array}{ll}
I_{n} & 0
\end{array}\right]^{H} T_{3}\right)=d_{0}^{(1)}+s_{0}^{(M C)}+e_{1}=d_{\mu}^{(1)},
$$

and further choosing $Z_{1}$ such that $Z_{2} Z_{1}=\left[\Pi_{1}^{H}, \Pi_{2}^{H}, \Pi_{8}^{H}, \Pi_{10}^{H}\right]$, we have

$$
\operatorname{rank}\left(Z_{1}^{H} Z_{2}^{H} \mathcal{L}_{1}\right)=d_{\mu}^{(1)}
$$

If we choose $T_{2}$ such that $T_{3} T_{2}=\left[\Theta_{5,2}, \Theta_{10,2}\right]$, then we have

$$
Z_{1}^{H} Z_{2}^{H} \mathcal{L}_{1}\left[\begin{array}{ll}
I_{n} & 0
\end{array}\right]^{H} T_{3} T_{2}=0
$$

and

$$
\operatorname{rank}\left(M T_{3} T_{2}\right)=d_{0}^{(2)}=d_{\mu}^{(2)}
$$

Finally, there exists a smooth matrix function $Z_{0}$ of size $\left(m, d_{\mu}^{(2)}\right)$ with orthonormal columns such that

$$
\operatorname{rank}\left(Z_{0}^{H} M\right)=d_{\mu}^{(2)}
$$

For s-index $\mu=2$, we have to consider the inflated triple

$$
\begin{aligned}
& \left(\tilde{\mathcal{M}}_{2}, \tilde{\mathcal{L}}_{2}, \tilde{\mathcal{N}}_{2}\right) \\
& =\left(\left[\begin{array}{ccc}
\tilde{M} & 0 & 0 \\
\dot{\tilde{M}}+\tilde{C} & \tilde{M} & 0 \\
\ddot{\tilde{M}}+2 \dot{\tilde{C}}+\tilde{K} & 2 \dot{\tilde{M}}+\tilde{C} & \tilde{M}
\end{array}\right],\left[\begin{array}{ccc}
\tilde{C} & 0 & 0 \\
\dot{\tilde{C}}+\tilde{K} & 0 & 0 \\
\ddot{C}+2 \dot{\tilde{K}} & 0 & 0
\end{array}\right],\left[\begin{array}{ccc}
\tilde{K} & 0 & 0 \\
\dot{\tilde{K}} & 0 & 0 \\
\ddot{\tilde{K}} & 0 & 0
\end{array}\right]\right) \text {. }
\end{aligned}
$$

Again, the identities in the diagonal blocks of $\tilde{\mathcal{M}}_{2}$ allow all the elimination of all other entries in the corresponding block rows of $\tilde{\mathcal{M}}_{2}$ without altering $\tilde{\mathcal{L}}_{2}$ or $\tilde{\mathcal{N}}_{2}$. Further eliminations using identity blocks in the global condensed form and block decompositions using local equivalence transformations yield a matrix triple of the 
Electronic Journal of Linear Algebra ISSN 1081-3810

A publication of the International Linear Algebra Society

Volume 22, pp. 310-347, March 2011

\section{ELA}

form

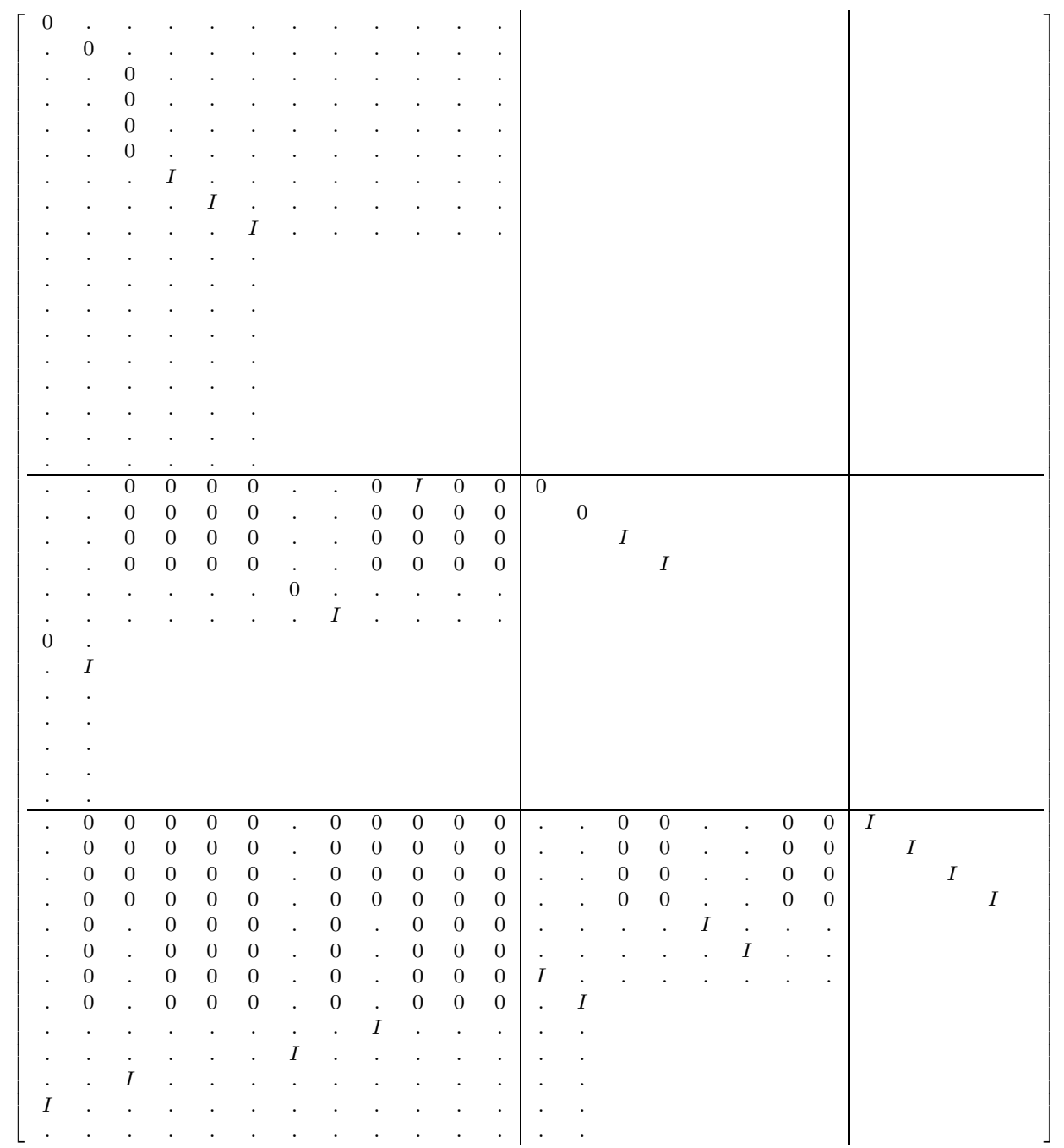




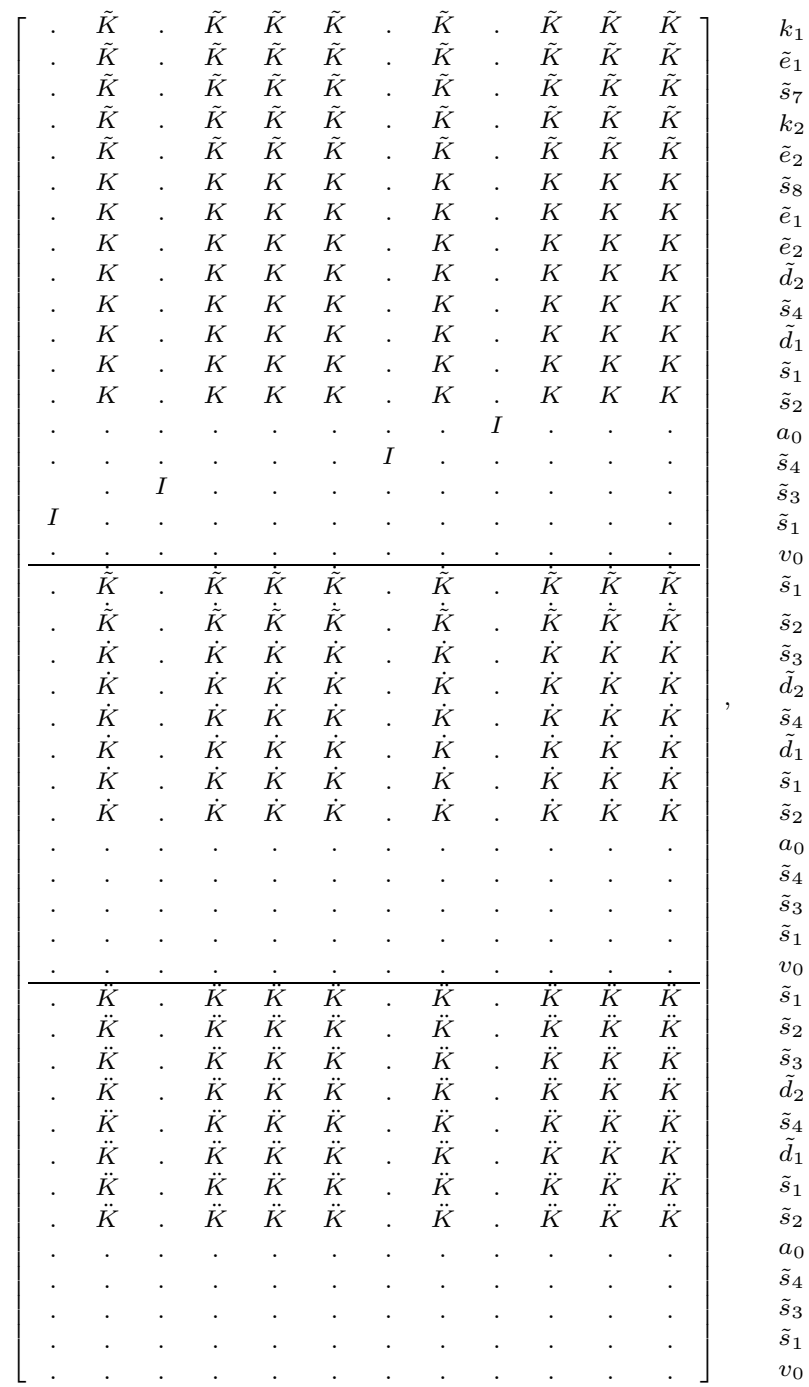

with dimensions $\tilde{e}_{2}=e_{2}-k_{2}, \tilde{s}_{7}=s_{0}^{(M C K)}+s_{0}^{(M C)}-e_{1}-e_{2}, \tilde{s}_{8}=s_{0}^{(M K)}-e_{1}+k_{1}-$ $e_{2}+k_{2}$. Thus, the triple $\left(\tilde{\mathcal{M}}_{2}, \tilde{\mathcal{L}}_{2}, \tilde{\mathcal{N}}_{2}\right)$ can be assumed to be in the form (B.3) and $\Pi$ and $\Theta$ can be partitioned into

$$
\Pi:=\left[\Pi_{1}^{H}, \ldots, \Pi_{44}^{H}\right]^{H}, \quad \Theta:=\left[\Theta_{1}, \ldots, \Theta_{28}\right]
$$

according to the block structure of (B.3). Then we have

$$
\begin{aligned}
{\left[\Pi_{1}^{H}, \ldots, \Pi_{6}^{H}, \Pi_{10}^{H}, \ldots, \Pi_{18}^{H}, \Pi_{20}^{H}, \Pi_{23}^{H}, \Pi_{25}^{H}, \Pi_{27}^{H}, \ldots, \Pi_{31}^{H}, \Pi_{44}^{H}\right]^{H} \mathcal{M}_{2}=0 } \\
\mathcal{M}_{2}\left[\Theta_{11}, \Theta_{12}, \Theta_{19}, \Theta_{20}, \Theta_{25}, \ldots, \Theta_{28}\right]=0
\end{aligned}
$$


Further decompositions of rows and columns of $\tilde{\mathcal{L}}_{2}$, where $\Pi_{\text {.,2 }}, \Theta_{\text {.,2 }}$ denote the parts of the rows and columns of $\tilde{\Pi}$ and $\tilde{\Theta}$, respectively, that correspond to null-rows or null-columns after the decomposition, yield

$$
\begin{aligned}
{\left[\Pi_{3}^{H}, \Pi_{6}^{H}, \Pi_{10}^{H}, \Pi_{12}^{H}, \Pi_{14}^{H}, \ldots, \Pi_{18}^{H}, \Pi_{20,2}^{H}, \Pi_{23,2}^{H}, \Pi_{25,2}^{H}, \Pi_{31}^{H}, \Pi_{44}^{H}\right]^{H} \mathcal{L}_{2}=0 } \\
Z^{H} \mathcal{L}_{2}\left[\Theta_{12,2}, \Theta_{19}, \Theta_{20}, \Theta_{25}, \ldots, \Theta_{28}\right]=0
\end{aligned}
$$

with

$$
Z:=\left[\Pi_{1}^{H}, \ldots, \Pi_{6}^{H}, \Pi_{10}^{H}, \ldots, \Pi_{18}^{H}, \Pi_{20}^{H}, \Pi_{23}^{H}, \Pi_{25}^{H}, \Pi_{27}^{H}, \ldots, \Pi_{31}^{H}, \Pi_{44}^{H}\right]
$$

and by setting

$$
\begin{aligned}
Z_{4} & =\left[\Pi_{3,2}^{H}, \Pi_{6,2}^{H}, \Pi_{10,2}^{H}, \Pi_{12,2}^{H}, \Pi_{18}^{H}, \Pi_{20,3}^{H}, \Pi_{23,3}^{H}, \Pi_{25,3}^{H}, \Pi_{31}^{H}, \Pi_{44}^{H}\right], \\
Z_{3} & =\left[\Pi_{3,1}^{H}, \Pi_{6,1}^{H}, \Pi_{10,1}^{H}, \Pi_{12,1}^{H}, \Pi_{14}^{H}, \ldots, \Pi_{17}^{H}, \Pi_{20,2}^{H}, \Pi_{23,2}^{H}, \Pi_{25,2}^{H}\right], \\
Z_{2} & =\left[\Pi_{1}^{H}, \Pi_{2}^{H}, \Pi_{4}^{H}, \Pi_{5}^{H}, \Pi_{11}^{H}, \Pi_{13}^{H}, \Pi_{20,1}^{H}, \Pi_{23,1}^{H}, \Pi_{25,1}^{H}, \Pi_{27}^{H}, \ldots, \Pi_{30}^{H}\right],
\end{aligned}
$$

we have

$$
\begin{aligned}
& \operatorname{rank}\left(Z_{3}^{H} \mathcal{N}_{2}\right)=c_{0}+c_{1}+c_{2}=a_{\mu}, \\
& \operatorname{rank}\left(Z_{2}^{H} \mathcal{L}_{2}\right)=e_{1}+e_{2}+d_{0}^{(1)}+s_{0}^{(M C)}+c_{0}+b_{1}=d_{\mu}^{(1)}+c_{0}+c_{1} .
\end{aligned}
$$

Furthermore, setting $T_{3}=\left[\Theta_{2,2}, \Theta_{4,2}, \Theta_{5,2}, \Theta_{6,2}, \Theta_{8,2}, \Theta_{10,2}, \Theta_{11,2}, \Theta_{12,2}\right]$ yields

$$
Z_{3}^{H} \mathcal{N}_{2}\left[\begin{array}{lll}
I_{n} & 0 & 0
\end{array}\right]^{H} T_{3}=0
$$

and we get

$$
\operatorname{rank}\left(Z_{2}^{H} \mathcal{L}_{2}\left[\begin{array}{ccc}
I_{n} & 0 & 0
\end{array}\right]^{H} T_{3}\right)=d_{2}^{(1)} .
$$

By choosing $Z_{1}$ such that $Z_{2} Z_{1}=\left[\Pi_{1}^{H}, \Pi_{2}^{H}, \Pi_{4}^{H}, \Pi_{5}^{H}, \Pi_{11}^{H}, \Pi_{13}^{H}\right]$, we have

$$
\operatorname{rank}\left(Z_{1}^{H} Z_{2}^{H} \mathcal{L}_{2}\right)=d_{\mu}^{(1)},
$$

and if we choose $T_{2}$ such that $T_{3} T_{2}=\left[\Theta_{5,2}, \Theta_{10,2}\right]$, then we have

$$
Z_{1}^{H} Z_{2}^{H} \mathcal{L}_{2}\left[\begin{array}{lll}
I_{n} & 0 & 0
\end{array}\right]^{H} T_{3} T_{2}=0
$$

as well as

$$
\operatorname{rank}\left(M T_{3} T_{2}\right)=d_{\mu}^{(2)} .
$$

Again, there exists a smooth matrix function $Z_{0}$ of size $\left(m, d_{\mu}^{(2)}\right)$ with orthonormal columns such that

$$
\operatorname{rank}\left(Z_{0}^{H} M\right)=d_{\mu}^{(2)}
$$

